\title{
GEOMETRIC QUANTITIES OF MANIFOLDS WITH GRASSMANN STRUCTURE
}

\author{
N. BOKAN*, P. MATZEU AND Z. RAKIĆ*
}

\begin{abstract}
We study geometry of manifolds endowed with a Grassmann structure which depends on symmetries of their curvature. Due to this reason a complete decomposition of the space of curvature tensors over tensor product vector spaces into simple modules under the action of the group $G=$ $G L(p, \mathbb{R}) \otimes G L(q, \mathbb{R})$ is given. The dimensions of the simple submodules, the highest weights and some projections are determined. New torsion-free connections on Grassmann manifolds apart from previously known examples are given. We use algebraic results to reveal obstructions to the existence of corresponding connections compatible with some type of normalizations and to enlighten previously known results from another point of view.
\end{abstract}

\section{$\S 1$. Introduction}

Let $\left(M^{n}, D\right)$ be a manifold with torsion-free connection $D, \mathfrak{g} \subseteq \mathfrak{g l}(V)$ the holonomy Lie algebra of $D$ at $m \in M$ and $V=T_{m} M$ the tangent space of $M$ at $m$. Then the curvature tensor $R$ of $D$ at a point $m \in M$ is a $\mathfrak{g}$-valued 2-form on $V$ which satisfies the Bianchi identity. One can introduce the structure of vector space in the set of these curvature ten-

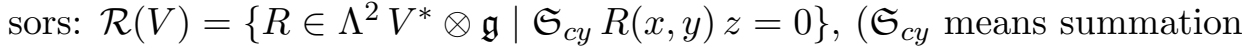
over all cyclic permutations of $(x, y, z))$. The decomposition of the space $\mathcal{R}(V)$ under the action of $\mathfrak{g}$ into simple $\mathfrak{g}$-submodules is important, because it carries some information about this manifold. The decomposition of the space $\mathcal{R}(V)$ for all holonomy groups of Levi-Civita connection was described by Alekseevsky [2]. For example, in the case of Levi-Civita connection $D=\nabla^{g}$ of a metric $g$ with holonomy group $S O(V)$ the irreducible components of $\mathcal{R}(V)$ correspond to the scalar curvature, traceless Ricci curvature and the Weyl tensor of $D=\nabla^{g}$. Vanishing of some of those irreducible components implies the system of differential equations of first

Received November 19, 2003.

Revised November 8, 2004.

2000 Mathematics Subject Classification: 53C30, $22 \mathrm{E} 45$.

${ }^{*}$ Research partially supported by the Ministry of Science of Serbia, project MM1646. 
order on connection $D$. Consequently these components give the first order invariants of $D$. For the group $G L(n, \mathbb{R})$ the complete decomposition of $\mathcal{R}(V)$ was given by Strichartz [47], for $S p(1) \otimes G L(n, \mathbb{H})$ (the full holonomy group of a quaternionic connection) it was given by Alekseevsky and Marchiafava [5], etc.

The main idea of this paper is to study geometry of manifolds endowed with a Grassmann structure which depends on symmetries of their curvature. More precisely we describe the decomposition of $\mathcal{R}(V)$ under the action of the group $G=G L(p, \mathbb{R}) \otimes G L(q, \mathbb{R})$ (or equivalently under the action of the Lie algebra $\mathfrak{g}=\mathfrak{g l}(p, \mathbb{R}) \otimes \mathbf{1}+\mathbf{1} \otimes \mathfrak{g l}(q, \mathbb{R}))$. We point out that this group is one of holonomy groups for torsion free connections in the Berger list [8]. We refer to [29] for more details about holonomy groups and [25], [14], [15], [39], [40] for special contribution to the classification of these groups.

Manifolds endowed with a Grassmann connection (called manifolds with Grassmann structure) have been studied by many authors and play an important role in modern geometry and physics (the Yang-Mills theory, the twistor theory, etc.).

The group $G$ and the associated geometric structure were studied by Akivis and Goldberg [1], Bailey and Eastwood [7], Machida and Sato [34], Manin [35], etc. The group $G$ as a significant example of a group of twistor type has been studied by Alekseevsky and Graev [4]. All these references announce an important role of this group in the twistor approach, a distinguished tool in modern geometry and mathematical physics (for more details we refer to [4], [42], [6], [18], etc.). Some applications of Grassmann structures, as well as other Segre structures to high energy physics were discussed in [36], [38], [3], etc. Among distinguished examples of manifolds with Grassmann structure are Grassmann manifolds $\mathcal{G} r\left(V^{p} \otimes V^{q}\right)=G L(p+q) / G L(p) \otimes G L(q)$. Since we deal with the general linear groups (and their Lie algebras) over $\mathbb{R}$, we simply write $G L(p)=G L(p, \mathbb{R})(\mathfrak{g l}(p, \mathbb{R})=\mathfrak{g l}(p))$. We use the notation $V^{p} \otimes V^{q}=V^{p \otimes q}$. The geometry and topology of these manifolds have been studied from several point of view by many authors (for more details see for example [1], [53], [22], [23], [16], [37], [48], [43], [32], [11], etc.). Other non-trivial examples of manifolds with Grassmann structures one can find for example in [3], [34], where the authors have considered also various connections over these manifolds and their applications to the complexification of hyper-Kähler manifolds, the tautological distributions, etc. 
In our research we reveal that the curvature related to a torsion-free connection of $\mathcal{G} r\left(V^{p \otimes q}\right)$ has a more complicated algebraic structure than in the case of an arbitrary smooth manifold whose structure group is $G L(p)$. This also implies a more rich geometry. We illustrate it by means of suitable examples of invariants, which did not appear in considering $G L(p)$.

The paper consists five sections. Apart from the first one, devoted to the history of the problem and to the announcement of some of our results, in Section 2 we report the known results we use in the paper. In Section 3 we prove that $\mathcal{R}(V)$ has 33 irreducible components under the action of the group $G$ (see Theorem 3.3). Since the highest weight vectors parametrize the irreducible components we consider them in Section 4, as well as the corresponding dimensions of irreducible components. Section 5 consists six subsections, each of them is devoted to various geometrical aspects of our algebraic results in the setting of geometry of manifolds with Grassmann structures and especially Grassmann manifolds. So, Subsection 5.1 is devoted to studying of geometry of manifolds endowed with half-flat connection. We consider projective invariants in Subsection 5.2 as well as the projections of a curvature $R$ into irreducible components that are not projectively invariant. We state that projectively invariant submodule consists of 29 irreducible components. This is an algebraically more complicated case in comparison with case of the action of group $G L(n)$ (see for example [46]). Anyhow, we want to emphasize (see Proposition 5.7) that a Leichtweiss connection has the Weyl projective curvature tensor which belongs to an irreducible component of $\mathcal{R}\left(\mathcal{G} r\left(V^{p \otimes q}\right)\right)$ for $p=q$, what is not true in the general case. Various normalizations of the Grassmann manifolds and algebraic structure of corresponding vector bundles of curvatures are studied in Subsection 5.3. This allows another approach to some known results as well as to obtain new results in this setting, related to obstructions of the existence of some type of normalizations. Subsection 5.4 deals with known and new examples of connections whose Ricci traces are symmetric and their curvatures belong to sum components in $\mathcal{R}\left(\mathcal{G} r\left(V^{p \otimes q}\right)\right)$. Ricci flat connections are studied in Subsection 5.5. Finally, in Subsection 5.6 we construct an example of torsion-free connection with the skew-symmetric Ricci tensor. These connections play an important role in studying absolute parallelizability of directions. All of these results related to any point or domain in $\mathcal{G} r\left(V^{p \otimes q}\right)$ makes also sense for space forms of Grassmann manifold (we refer to [52] for more details). 


\section{§2. Preliminaries}

Let us recall that the study of geometry and topology by using the action of some group on the vector space of some geometric quantities was initiated by Alekseevsky [2], Singer and Thorpe [46], Kulkarni [31] and many other authors who applied this method to the analysis of several geometric problems (see for example [20], [50], and [13], [44] for more details).

In this section we introduce some basic notions and notations we need in the following sections, as well as some known results. All vector spaces and general linear groups we consider in this paper are taken over $\mathbb{R}$. Let $\mathfrak{S}_{r}$ denotes the symmetric group in $r$ letters and $\mathfrak{A}_{r}$ its subgroup of even permutations. Let $V^{p}$ be a real vector space of dimension $p$ and $V=$ $V^{p \otimes q}=V^{p} \otimes V^{q}$ be a tensor product of real vector spaces. If $\left(e_{i}\right)$ and $\left(f_{\alpha}\right)$ are basis in $V^{p}$ and $V^{q}$ respectively, then $\left(v_{i \alpha}=e_{i} \otimes f_{\alpha}\right)$ is the standard basis in $V^{p \otimes q}$. We denote the vectors of the dual basis by adding a ${ }^{*}$, to the vectors of such basis. We denote the components of an arbitrary vector $v$ in $V^{p \otimes q}$ by $v_{(i \alpha)}$. Hence a tensor of rank $(k, r)$ has the components $T_{\left(i_{1} \alpha_{1}\right)\left(i_{2} \alpha_{2}\right) \cdots\left(i_{r} \alpha_{r}\right)}^{\left(j_{1} \beta_{1}\right)}$.

We denote the vector space of tensors of rank $(k, r)$ by $\mathcal{T}_{r}^{k}$. If some of $k$ or $r$ is equal to zero then we drop the corresponding index. Moreover, if $k=0$ we denote it by $V^{\otimes r}$. There is a natural action of the group $\mathfrak{S}_{r}$ on a tensor of $\operatorname{rank}(0, r)$. For $\sigma \in \mathfrak{S}_{r}$ we have

$$
\sigma \cdot T_{\left(i_{1} \alpha_{1}\right)\left(i_{2} \alpha_{2}\right) \cdots\left(i_{r} \alpha_{r}\right)}=T_{\left(i_{\sigma(1)} \alpha_{\sigma(1)}\right)\left(i_{\sigma(2)} \alpha_{\sigma(2)}\right) \cdots\left(i_{\sigma(r)} \alpha_{\sigma(r)}\right)} .
$$

Let us remark that this action will be considered as the action of the group ring of the symmetric group, i.e. $\mathfrak{G}\left(\mathfrak{S}_{r}\right)$.

The group $G L(p) \otimes G L(q)$ is a subgroup of $G L(p q)$ and it acts on $V^{p} \otimes V^{q}$ such that $G L(p)$ acts on $V^{p}$ in the usual way and $G L(q)$ acts on $V^{q}$ by inverse from the right. There is the natural projection

$$
\xi: G L(p) \times G L(q) \longrightarrow G L(p) \otimes G L(q)
$$

that defines a fibre bundle with fibre $\mathbb{R}^{*}$ by the scalar multiplication and the dimension of the group $G L(p) \otimes G L(q)$ is $p^{2}+q^{2}-1$ (see [34]).

Let $R \in \mathcal{T}_{3}^{1}$. Then $R$ is an algebraic curvature tensor if it satisfies the 
following symmetry conditions:

$$
\begin{gathered}
\sigma_{12} \cdot R\left(x_{1}, x_{2}, x_{3}, \omega\right)=R\left(x_{2}, x_{1}, x_{3}, \omega\right)=-R\left(x_{1}, x_{2}, x_{3}, \omega\right), \\
\sum_{\sigma \in \mathfrak{A}_{3}} R\left(x_{\sigma(1)}, x_{\sigma(2)}\right) x_{\sigma(3)}=\mathfrak{A}_{3}^{+} \cdot R\left(x_{1}, x_{2}\right) x_{3}=0,
\end{gathered}
$$

where $\sigma_{12}$ is the transposition (12), $\mathfrak{A}_{3}^{+}=\sum_{\sigma \in \mathfrak{A}_{3}} \sigma$ is considered as an element of the group ring $\mathfrak{G}\left(\mathfrak{S}_{3}\right)$, and

$$
R\left(x_{1}, x_{2}, x_{3}, \omega\right)=\omega\left(R\left(x_{1}, x_{2}\right) x_{3}\right)=\left\langle R\left(x_{1}, x_{2}\right) x_{3}, \omega\right\rangle .
$$

It is clear that algebraic curvature tensors build a vector subspace $\mathcal{R}\left(V^{n}\right)$ in $\mathcal{T}_{3}^{1}$. The Ricci traces, $\rho^{i}: \mathcal{T}_{3}^{1} \rightarrow \mathcal{T}_{2}$, are defined as follows

$$
\rho^{i}=\operatorname{con}(i, 4) R, \quad i=1,2,3,
$$

where $\operatorname{con}(i, 4)$ means contraction in the $i^{\text {th }}$ covariant and contravariant indices. It is easy to see the Ricci traces $\rho^{2}$ and $\rho^{3}$ can be expressed in terms of $\rho^{1}$, for $R \in \mathcal{R}\left(V^{n}\right)$.

In the following, we need a fundamental result obtained by Strichartz in $[47]$.

The group $G L(n)$ acts naturally on $\mathcal{T}_{3}^{1}$ by

$$
\pi(g) R\left(x_{1}, x_{2}, x_{3}, \omega\right)=R\left(g^{-1} x_{1}, g^{-1} x_{2}, g^{-1} x_{3}, g^{\tau} \omega\right),
$$

and this action defines on $\mathcal{T}_{3}^{1}$ the structure of $G L(n)$-module. As it is known, the simple $G L(n)$-modules are parameterized by the lattice of highest weights. We denote by $\pi(m)$ the simple $G L(n)$-module of the highest weight $m=\left(m_{1}, m_{2}, \ldots, m_{n}\right)\left(m_{1} \geq m_{2} \geq \cdots \geq m_{n}, m_{i}\right.$ are integers). Following a standard notation we delete the string of zeros, so that $\pi(2,-1)$ means $\pi(2,0, \ldots, 0,-1)$. It is clear that $\pi(1)$ is the fundamental representation of $G L(n)$ in $V^{n}$ and $\pi(-1)$ is its contragredient representation in $\left(V^{n}\right)^{*}$. In [47] the following theorem has been shown.

THEOREM 2.1. The semi-simple $G L(n)$-module $\mathcal{R}\left(V^{n}\right)$ is the direct sum of simple $G L(n)$-modules,

$$
\mathcal{R}\left(V^{n}\right)=\pi(2) \oplus \pi(1,1) \oplus \pi(2,1,-1),
$$

(when $n=2$ the third component is deleted).

In the same theorem he found corresponding projections on irreducible components. 


\section{§3. Decomposition of $\mathcal{R}(V)$}

Since we are interested in the vector space of curvature tensors over tensor product vector spaces, we define a natural generalization of the action of the group $G$.

Let $\triangle$ be the diagonal mapping of $\mathfrak{G}\left(\mathfrak{S}_{r}\right)$ to $\mathfrak{G}\left(\mathfrak{S}_{r}\right) \otimes \mathfrak{G}\left(\mathfrak{S}_{r}\right)$, i.e.,

$$
\triangle: \mathfrak{G}\left(\mathfrak{S}_{r}\right) \longrightarrow \mathfrak{G}\left(\mathfrak{S}_{r}\right) \otimes \mathfrak{G}\left(\mathfrak{S}_{r}\right),
$$

given by the following formula $\triangle(\sigma)=\sigma \otimes \sigma$ and extended by linearity. We consider the action on $V^{\otimes r}\left(V=V^{p} \otimes V^{q}\right)$

$$
\left(\sigma_{1} \otimes \sigma_{2}\right) \cdot T\left(x_{1} \otimes y_{1}, \ldots, x_{r} \otimes y_{r}\right)=T\left(x_{\sigma_{1}(1)} \otimes y_{\sigma_{2}(1)}, \ldots, x_{\sigma_{1}(r)} \otimes y_{\sigma_{2}(r)}\right) .
$$

Then the formulas (1) and (2) have to be obviously modified as

$$
\begin{gathered}
\triangle\left(\sigma_{12}\right) . R\left(x_{1} \otimes y_{1}, x_{2} \otimes y_{2}, x_{3} \otimes y_{3}, \omega \otimes \theta\right) \\
\quad=-R\left(x_{1} \otimes y_{1}, x_{2} \otimes y_{2}, x_{3} \otimes y_{3}, \omega \otimes \theta\right) \\
\triangle\left(\mathfrak{A}_{3}^{+}\right) . R\left(x_{1} \otimes y_{1}, x_{2} \otimes y_{2}\right) x_{3} \otimes y_{3}=0 .
\end{gathered}
$$

Consequently the Ricci traces of the tensors of rank $(1,3)$, become

$$
\rho^{i \alpha}=\operatorname{con}((i, \alpha)(4,4)) R=\overline{\operatorname{con}}(i, 4) \circ \overline{\operatorname{con}}(\alpha, 4) R,
$$

where $\overline{c o n}(i, 4)=\operatorname{con}(i, 4) \otimes i d$ and $\overline{c o n}(\alpha, 4)=i d \otimes \operatorname{con}(\alpha, 4)$.

We use the formulae (1), (7) and (8), to prove the following proposition.

Proposition 3.1. The vector space of Ricci traces is spanned by $\rho^{11}$, $\rho^{12}$ and $\rho^{13}$.

This proposition is very important since the kernels and images of Ricci traces are submodules of $G$-module $\mathcal{R}(V)$.

In the rest of the paper we prefer the terminology of modules (simple and semi-simple submodules) rather then the terminology of representations (irreducible, reducible subrepresentations).

Since the group $G$ is a subgroup of $G L(p q)$ and because of the decomposition of $\mathcal{R}\left(V^{p q}\right)$ given in Theorem 2.1, it follows that its simple modules are just stable under the action of the group $G$. Our next task is finding their decomposition into simple parts. So, let us denote by $\Pi_{1}(m)$ and $\Pi_{2}(m)$ the simple $G L(p)$ and $G L(q)$-modules respectively, where $m$ is, as before, the highest weight. 
The tensors that belong to the component $\pi^{a}(2,1)$ are the only ones which satisfy both conditions (1) and (2). Consequently, making tensor product of the component $\pi^{a}(2,1)$ by $\pi(-1)$, we obtain the decomposition of the space of the curvature tensors into simple modules under the action of the group $G L(V)$, that is practically the proof of Theorem 2.1. We apply this idea on $V=V^{p} \otimes V^{q}$.

Firstly, one can use the action of the group $G$ on the vector space $V \otimes V$ to check the following lemma (see [34], [26]).

LEMMA 3.2. Let $V=V^{p} \otimes V^{q}$. Under the action of the group $G L(p) \otimes$ $G L(q)$ the space $V \otimes V$ is decomposed as follows

(i1) $\pi(2)=\Pi_{1}(2) \otimes \Pi_{2}(2) \oplus \Pi_{1}(1,1) \otimes \Pi_{2}(1,1)$,

$(i 2) \pi(1,1)=\Pi_{1}(2) \otimes \Pi_{2}(1,1) \oplus \Pi_{1}(1,1) \otimes \Pi_{2}(2)$,

where the corresponding Young symmetrizers are

$(c 1) \bar{c}_{(2)}=c_{(2)} \otimes c_{(2)}+c_{(1,1)} \otimes c_{(1,1)}$,

$(c 2) \bar{c}_{(1,1)}=c_{(2)} \otimes c_{(1,1)}+c_{(1,1)} \otimes c_{(2)}$.

Since we need the decomposition of $G L(p q)$-module $\pi(2,1,-1)$ into direct sum of simple $G$-modules, we consider the space $(V \wedge V) \otimes V$, and use the Young symmetrizers. Firstly, let us recall for $i=p, j=1$, or $i=q$, $j=2$, that

$$
V^{i} \otimes V^{i} \otimes V^{i}=\Pi_{j}(3) \oplus \Pi_{j}^{s}(2,1) \oplus \Pi_{j}^{a}(2,1) \oplus \Pi_{j}(1,1,1)
$$

where $\Pi_{j}^{s}(2,1)$ consists of tensors satisfying the first Bianchi identity and symmetry in first two places.

If we put $\mathfrak{B}=3 I-\mathfrak{A}_{3}^{+}$, then we have the following symmetrizers of $\left(V^{i}\right)^{\otimes 3}$ :

$$
\begin{aligned}
c_{(3)} & =\frac{1}{6}\left(\mathfrak{A}_{3}^{+}+\sigma_{12} \mathfrak{A}_{3}^{+}\right), & c_{(1,1,1)} & =\frac{1}{6}\left(\mathfrak{A}_{3}^{+}-\sigma_{12} \mathfrak{A}_{3}^{+}\right), \\
c_{(2,1)}^{a} & =\frac{1}{6}\left(\mathfrak{B}-\sigma_{12} \mathfrak{B}\right), & c_{(2,1)}^{s} & =\frac{1}{6}\left(\mathfrak{B}+\sigma_{12} \mathfrak{B}\right) .
\end{aligned}
$$

Making tensor product of the corresponding components, one can find the symmetrizers of the simple $G$-modules of $V^{\otimes 3}$. More precisely, we have the 
following decomposition of $\pi^{a}(2,1)$ in the terms of the corresponding Young symmetrizers

$$
\begin{aligned}
c_{(3)} \otimes c_{(2,1)}^{a}= & \frac{1}{36}\left(\mathfrak{A}_{3}^{+} \otimes \mathfrak{B}-\mathfrak{A}_{3}^{+} \otimes \sigma_{12} \mathfrak{B}+\sigma_{12} \mathfrak{A}_{3}^{+} \otimes \mathfrak{B}-\sigma_{12} \mathfrak{A}_{3}^{+} \otimes \sigma_{12} \mathfrak{B}\right), \\
c_{(2,1)}^{a} \otimes c_{(3)}= & \frac{1}{36}\left(\mathfrak{B} \otimes \mathfrak{A}_{3}^{+}-\sigma_{12} \mathfrak{B} \otimes \mathfrak{A}_{3}^{+}+\mathfrak{B} \otimes \sigma_{12} \mathfrak{A}_{3}^{+}-\sigma_{12} \mathfrak{B} \otimes \sigma_{12} \mathfrak{A}_{3}^{+}\right), \\
c_{(1,1,1)} \otimes c_{(2,1)}^{s}= & \frac{1}{36}\left(\mathfrak{A}_{3}^{+} \otimes \mathfrak{B}-\sigma_{12} \mathfrak{A}_{3}^{+} \otimes \mathfrak{B}+\mathfrak{A}_{3}^{+} \otimes \sigma_{12} \mathfrak{B}-\sigma_{12} \mathfrak{A}_{3}^{+} \otimes \sigma_{12} \mathfrak{B}\right), \\
c_{(2,1)}^{s} \otimes c_{(1,1,1)}= & \frac{1}{36}\left(\mathfrak{B} \otimes \mathfrak{A}_{3}^{+}+\sigma_{12} \mathfrak{B} \otimes \mathfrak{A}_{3}^{+}-\mathfrak{B} \otimes \sigma_{12} \mathfrak{A}_{3}^{+}-\sigma_{12} \mathfrak{B} \otimes \sigma_{12} \mathfrak{A}_{3}^{+}\right), \\
(11) \quad\left(c_{(2,1)} \otimes c_{(2,1)}\right)^{a}=\Delta\left(c_{(2,1)}^{a}\right)- & \left(c_{(3)} \otimes c_{(2,1)}^{a}+c_{(2,1)}^{a} \otimes c_{(3)}\right. \\
& \left.+c_{(1,1,1)} \otimes c_{(2,1)}^{s}+c_{(2,1)}^{s} \otimes c_{(1,1,1)}\right) .
\end{aligned}
$$

The corresponding decomposition of the component $\pi^{a}(2,1)$ in terms of the highest weights is

$$
\begin{gathered}
\pi^{a}(2,1)=\Pi_{1}(3) \otimes \Pi_{2}^{a}(2,1) \oplus \Pi_{1}^{a}(2,1) \otimes \Pi_{2}(3) \oplus \Pi_{1}(2,1) \otimes \Pi_{2}(2,1) \\
\oplus \Pi_{1}^{s}(2,1) \otimes \Pi_{2}(1,1,1) \oplus \Pi_{1}(1,1,1) \otimes \Pi_{2}^{s}(2,1) .
\end{gathered}
$$

It is clear that all simple $G$-modules given in (12) satisfy all needed symmetry conditions, and making tensor product of them by $V^{*}=\pi(-1)=$ $\Pi_{1}(-1) \otimes \Pi_{2}(-1)$, we obtain the decomposition of the space of curvature tensors into simple $G$-modules (using the Littlewood-Richardson rule [34, p. 331], see also [19], [12]).

Let we introduce symmetric analogue of the curvature tensor (symmetry in the first two indices and the first Bianchi identity) by

$$
\mathcal{R}^{*}\left(V^{n}\right)=\pi^{s}(2) \oplus \pi^{s}(1,1) \oplus \pi^{s}(2,1,-1),
$$

We summarize the previous considerations in the following theorem.

TheOREm 3.3. Let $G=G L(p) \otimes G L(q)$ and $V=V^{p \otimes q}=V^{p} \otimes V^{q}$ then the space of curvature tensors $\mathcal{R}(V)$ decomposes as follows $(p, q>3)$

$$
\begin{aligned}
\mathcal{R}(V)=\Pi_{1}(2) \otimes \Pi_{2}(2) \oplus \Pi_{1}(1,1) \otimes \Pi_{2}(1,1) \\
\oplus \Pi_{1}(2) \otimes \Pi_{2}(1,1) \oplus \Pi_{1}(1,1) \otimes \Pi_{2}(2) \\
\oplus\left(\Pi_{1}^{t s}(2) \oplus \Pi_{1}(3,-1)\right) \otimes \mathcal{R}\left(V^{q}\right) \\
\oplus \mathcal{R}\left(V^{p}\right) \otimes\left(\Pi_{2}^{t s}(2) \oplus \Pi_{2}(3,-1)\right)
\end{aligned}
$$




$$
\begin{aligned}
& \oplus\left(\Pi_{1}^{t a}(1,1) \oplus \Pi_{1}(1,1,1,-1)\right) \otimes \mathcal{R}^{*}\left(V^{q}\right) \\
& \oplus \mathcal{R}^{*}\left(V^{p}\right) \otimes\left(\Pi_{2}^{t a}(1,1) \oplus \Pi_{2}(1,1,1,-1)\right) \\
& \oplus \Pi_{1}(2,1,-1) \otimes \Pi_{2}(2) \oplus \Pi_{1}(2) \otimes \Pi_{2}(2,1,-1) \\
& \oplus \Pi_{1}(2,1,-1) \otimes \Pi_{2}(1,1) \oplus \Pi_{1}(1,1) \otimes \Pi_{2}(2,1,-1) \\
& \oplus \Pi_{1}(2,1,-1) \otimes \Pi_{2}(2,1,-1) .
\end{aligned}
$$

The superscripts ' $t s$, and ' $t a$ ' indicate that those components consist of totally symmetric and totally skew-symmetric tensors in covariant places.

Remark 3.4. Some components in the decomposition in Theorem 3.3 for $p, q \leq 3$ vanish since their dimensions are equal to zero (see Table (dimension) in the next section). In Section 5, we present various projection operators only for $p, q>3$, because of the same reason.

Remark 3.5. Let us also remark, that in this decomposition there exist four simple modules of multiplicity 3 , four simple modules of multiplicity 2 and 13 simple modules of multiplicity 1.

\section{§4. Simple modules their dimensions and highest weights}

The main purpose of this section is to obtain the highest weight vectors of all $G$-modules which appear in the decomposition of $\mathcal{R}(V)$ (stated in Theorem 3.3). We reach these results by studying all simple modules of $G L(n)$.

In order to know the dimensions of the simple $G$-modules, we need to compute the dimensions of the simple $G L(n)$-modules enlarging the result of Theorem 2.1. More precisely, we have Table 1.

Finally, by a straightforward computation, with use of Theorem 3.3 and Table 1 we get

$$
\operatorname{dim} \mathcal{R}(V)=\frac{p^{2} q^{2}\left(p^{2} q^{2}-1\right)}{3} .
$$

If we use the following notations:

$$
\begin{aligned}
& v_{\left(i_{1} \alpha_{1}\right) \cdots\left(i_{k} \alpha_{1}\right)\left(j_{1} \beta_{1}\right)^{*} \cdots\left(j_{l} \beta_{l}\right)^{*}} \\
& \quad=\left(e_{i_{1}} \otimes f_{\alpha_{1}}\right) \cdots\left(e_{i_{k}} \otimes f_{\alpha_{k}}\right) \otimes\left(e_{j_{1}}^{*} \otimes f_{\beta_{1}}^{*}\right) \cdots\left(e_{j_{l}}^{*} \otimes f_{\beta_{l}}^{*}\right) \\
& \cong\left(e_{i_{1}} \otimes \cdots \otimes e_{i_{k}} \otimes e_{j_{1}}^{*} \otimes \cdots \otimes e_{j_{l}}^{*}\right) \\
& \quad \otimes\left(f_{j_{1}} \otimes \cdots \otimes f_{j_{k}} \otimes f_{\beta_{1}}^{*} \otimes \cdots \otimes f_{\beta_{l}}^{*}\right)
\end{aligned}
$$




\begin{tabular}{ccc}
\hline \hline Component & Dimension & Highest Weight Vector \\
\hline$\Pi^{a}(2)$ & $\frac{1}{2} n(n+1)$ & $\sum_{k}\left(e_{1} \wedge e_{k}\right) \otimes e_{1} \otimes e_{k}^{*}$ \\
$\Pi^{a}(1,1)$ & $\frac{1}{2} n(n-1)$ & $\sum_{k}\left(\left(e_{1} \wedge e_{2}\right) \otimes e_{k}\right.$ \\
$\Pi^{a}(2,1,-1)$ & $\frac{1}{3} n^{2}\left(n^{2}-4\right)$ & $\left.+\left(e_{k} \wedge e_{2}\right) \otimes e_{1}\right) \otimes e_{k}^{*}$ \\
$\Pi^{s}(2)$ & $\frac{1}{2} n(n+1)$ & $\sum_{k}\left(\left(e_{1} \circledast e_{2}\right) \otimes e_{1} \otimes e_{n}^{*}\right.$ \\
$\Pi^{s}(1,1)$ & $\frac{1}{2} n(n-1)$ & $\left.-e_{1} \otimes e_{1} \otimes e_{k}\right) \otimes e_{k}^{*}$ \\
$\Pi^{s}(2,1,-1)$ & $\frac{1}{3} n^{2}\left(n^{2}-4\right)$ & $\left(\left(e_{1} \circledast e_{2}\right) \otimes e_{1}\right.$ \\
$\Pi^{2}(3,-1)$ & $\frac{1}{6} n(n+3)\left(n^{2}-1\right)$ & $\left.-e_{1} \circledast e_{k}\right) \otimes e_{2}$ \\
$\Pi^{t s}(2)$ & $\frac{1}{2} n(n+1)$ & $e_{1} \otimes e_{1} \otimes e_{1} \otimes e_{n}^{*}$ \\
$\Pi^{t a}(1,1)$ & $\frac{1}{2} n(n-1)$ & $\left.\sum_{k}\left(e_{1} \circledast e_{1} \circledast e_{k}\right) \otimes e_{k}\right) \otimes e_{k}^{*}$ \\
$\Pi_{(1,1,1,-1)}$ & $\sum_{k}\left(e_{1} \wedge e_{2} \wedge e_{k}\right) \otimes e_{k}^{*}$ \\
\hline \hline
\end{tabular}

Table 1.

where $v_{(i \alpha)}=e_{i} \otimes f_{\alpha}$ is the standard basis of $V^{p} \otimes V^{q}$ and $v_{(j \beta)}^{*}=e_{j}^{*} \otimes f_{\beta}^{*}$ is the standard basis of $\left(V^{*}\right)^{p} \otimes\left(V^{*}\right)^{q}$, one can rewrite (13) as

$$
\begin{aligned}
& v_{\left(i_{1} \alpha_{1}\right) \cdots\left(i_{k} \alpha_{1}\right)\left(j_{1} \beta_{1}\right)^{*} \cdots\left(j_{l} \beta_{l}\right)^{*}} \\
& \quad=v_{\left(i_{1} \alpha_{1}\right)} \otimes \cdots \otimes v_{\left(i_{k} \alpha_{1}\right)} \otimes v_{\left(j_{1} \beta_{1}\right)}^{*} \otimes \cdots \otimes v_{\left(j_{l} \beta_{l}\right)}^{*} .
\end{aligned}
$$

One can obtain, by making tensor product of the appropriate highest weight vectors of components from previous Table, the highest weight vectors of simple $G$-modules of the following 24 components:

$$
\begin{aligned}
& \Pi_{1}(3,-1) \otimes \Pi_{2}^{a}(2), \quad \Pi_{1}^{a}(2) \otimes \Pi_{2}(3,-1), \\
& \Pi_{1}(3,-1) \otimes \Pi_{2}^{a}(1,1), \quad \Pi_{1}^{a}(1,1) \otimes \Pi_{2}(3,-1), \\
& \Pi_{1}(3,-1) \otimes \Pi_{2}^{a}(2,1,-1), \quad \Pi_{1}^{a}(2,1,-1) \otimes \Pi_{2}(3,-1), \\
& \Pi_{1}^{t s}(2) \otimes \Pi_{2}^{a}(2), \quad \Pi_{1}^{a}(2) \otimes \Pi_{2}^{t s}(2), \quad \Pi_{1}^{t s}(2) \otimes \Pi_{2}^{a}(1,1), \quad \Pi_{1}^{a}(1,1) \otimes \Pi_{2}^{t s}(2), \\
& \Pi_{1}^{t s}(2) \otimes \Pi_{2}^{a}(2,1,-1), \quad \Pi_{1}^{a}(2,1,-1) \otimes \Pi_{2}^{t s}(2),
\end{aligned}
$$




$$
\begin{aligned}
& \Pi_{1}(1,1,1,-1) \otimes \Pi_{2}^{s}(2), \quad \Pi_{1}^{s}(2) \otimes \Pi_{2}(1,1,1,-1), \\
& \Pi_{1}(1,1,1,-1) \otimes \Pi_{2}^{s}(1,1), \quad \Pi_{1}^{s}(1,1) \otimes \Pi_{2}(1,1,1,-1), \\
& \Pi_{1}^{t a}(1,1) \otimes \Pi_{2}^{s}(2,1,-1), \quad \Pi_{1}^{s}(2,1,-1) \otimes \Pi_{2}^{t a}(1,1), \\
& \Pi_{1}^{t a}(1,1) \otimes \Pi_{2}^{s}(2), \quad \Pi_{1}^{s}(2) \otimes \Pi_{2}^{t a}(1,1), \\
& \Pi_{1}^{t a}(1,1) \otimes \Pi_{2}^{s}(1,1), \quad \Pi_{1}^{s}(1,1) \otimes \Pi_{2}^{t a}(1,1), \\
& \Pi_{1}(1,1,1,-1) \otimes \Pi_{2}^{s}(2,1,-1), \quad \Pi_{1}^{s}(2,1,-1) \otimes \Pi_{2}(1,1,1,-1) .
\end{aligned}
$$

The highest weights of the residual 9 components are given in Table 2 .

\section{§5. Applications}

To apply the results in Sections 3 and 4 we introduce a notion of the Grassmann structure. A Grassmann structure of type $(p, q)$ on a manifold $M$ is, by definition, an isomorphism from the tangent bundle $T M$ of $M$ to the tensor product $E \otimes H$ of two vector bundles $E$ and $H$ with rank $p$ and $q$ over $M$ respectively $(T M \simeq E \otimes H)$. If $\operatorname{dim} M=n$, then $n=p q$. Typical examples of manifolds with Grassmann structures are the Grassmann manifolds $\mathcal{G} r\left(V^{p \otimes q}\right)$. The tangent bundle $T T M$ of $T M$ has a Grassmann structure of type $(n, 2)$. Let $F^{r} M$ be the $r$-frame bundle of $M$. The $n(r+1)$ dimensional manifold $F^{r} M$ has a Grassmann structure of type $(n, r+1)$. We refer to [3], [34] for more details.

A linear connection $D$ on $M$ is called a Grassmann connection if it preserves the Grassmann structure. This means that for any vector field $X$ on $M$ and local sections $e \in \Gamma(E)$ and $h \in \Gamma(H)$,

$$
D_{X}(e \otimes h)=D_{X}^{E} e \otimes h+e \otimes D_{X}^{H} h,
$$

where $D^{E}, D^{H}$ are connections in the bundles $E, H$ respectively. If $D$ is torsion-free, its holonomy group is $G=G L(p) \otimes G L(q)$.

Throughout this section we consider only smooth manifolds nevertheless many results can be stated in the setting of complex manifolds and holomorphic connections. We note that using analytic continuation any real analytic connection $D$ over a real analytic Grassmann manifold allows extension to a holomorphic connection $D^{\mathbb{C}}$ over a holomorphic Grassmann manifold and $D$ can be reconstructed from $D^{\mathbb{C}}$ in terms of some antiholomorphic involution.

Let $M$ be a real manifold with Grassmann structure of type $(p, q)(p q=$ $n$ ) and $D$ a torsion-free connection whose curvature tensor $R$ is given by

$$
R(X, Y)=\left[D_{X}, D_{Y}\right]-D_{[X, Y]}
$$




\begin{tabular}{|c|c|}
\hline Component & Highest Weight Vector \\
\hline$\Pi_{1}(2) \otimes \Pi_{2}(2)$ & $\begin{array}{l}\sum_{k, j}\left(v_{(11)(k j)(11)(k j)^{*}}-v_{(k j)(11)(11)(k j)^{*}}\right. \\
\quad-v_{(11)(k 1)(1 j)(k j)^{*}}+v_{(k 1)(11)(1 j)(k j)^{*}} \\
\left.\quad-v_{(11)(1 j)(k 1)(k j)^{*}}+v_{(1 j)(11)(k 1)(k j)^{*}}\right)\end{array}$ \\
\hline$\Pi_{1}(2) \otimes \Pi_{2}(2,1,-1)$ & $\begin{array}{l}\sum_{k}\left(v_{(11)(k 2)(11)(k q)^{*}}-v_{(k 2)(11)(11)(k q)^{*}}\right. \\
\quad-v_{(11)(k 1)(12)(k q)^{*}}+v_{(k 1)(11)(12)(k q)^{*}} \\
\quad-v_{(11)(12)(k 1)(k q)^{*}}+v_{(12)(11)(k 1)(k q)^{*}}\end{array}$ \\
\hline$\Pi_{1}(2,1,-1) \otimes \Pi_{2}(2)$ & $\begin{array}{l}\sum_{k}\left(v_{(11)(2 k)(11)(p k)^{*}}-v_{(2 k)(11)(11)(p k)^{*}}\right. \\
\quad-v_{(11)(21)(1 k)(p k)^{*}}+v_{(21)(11)(1 k)(p k)^{*}} \\
\quad-v_{(11)(1 k)(21)(p k)^{*}}+v_{(1 k)(11)(21)(p k)^{*}}\end{array}$ \\
\hline$\Pi_{1}(2) \otimes \Pi_{2}(1,1)$ & $\begin{array}{l}\sum_{k, j}\left(v_{(1 j)(k 2)(11)(k j)^{*}}-v_{(k 2)(1 j)(11)(k j)^{*}}\right. \\
\quad-v_{(1 j)(k 1)(12)(k j)^{*}}+v_{(k 1)(1 j)(12)(k j)^{*}} \\
\quad-v_{(1 j)(12)(k 1)(k j)^{*}}+v_{(12)(1 j)(k 1)(k j)^{*}} \\
\left.\quad+v_{(11)(k 2)(1 j)(k j)^{*}}-v_{(k 2)(11)(1 j)(k j)^{*}}\right)\end{array}$ \\
\hline$\Pi_{1}(1,1) \otimes \Pi_{2}(2)$ & $\begin{array}{l}\sum_{k, j}\left(v_{(k 1)(2 j)(11)(k j)^{*}}-v_{(2 j)(k 1)(11)(k j)^{*}}\right. \\
\quad+v_{(11)(2 j)(k 1)(k j)^{*}}-v_{(2 j)(11)(k 1)(k j)^{*}} \\
\quad-v_{(k 1)(21)(1 j)(k j)^{*}}+v_{(21)(k 1)(1 j)(k j)^{*}} \\
\left.\quad-v_{(k 1)(1 j)(21)(k j)^{*}}+v_{(1 j)(k 1)(21)(k j)^{*}}\right)\end{array}$ \\
\hline$\Pi_{1}(1,1) \otimes \Pi_{2}(1,1)$ & $\begin{array}{l}\sum_{k, j}\left(v_{(k j)(22)(11)(k j)^{*}}-v_{(22)(k j)(11)(k j)^{*}}\right. \\
\quad+v_{(11)(22)(k j)(k j)^{*}}-v_{(22)(11)(k j)(k j)^{*}} \\
\quad-v_{(k 1)(2 j)(12)(k j)^{*}}+v_{(2 j)(k 1)(12)(k j)^{*}} \\
\left.\quad-v_{(k 1)(12)(2 j)(k j)^{*}}+v_{(12)(k 1)(2 j)(k j)^{*}}\right)\end{array}$ \\
\hline$\Pi_{1}(1,1) \otimes \Pi_{2}(2,1,-1)$ & $\begin{aligned} \sum_{k} & \left(v_{(k 1)(22)(11)(k q)^{*}}-v_{(22)(k 1)(11)(k q)^{*}}\right. \\
& +v_{(11)(22)(k 1)(k q)^{*}}-v_{(22)(11)(k 1)(k q)^{*}} \\
& -v_{(k 1)(21)(12)(k q)^{*}}+v_{(21)(k 1)(12)(k q)^{*}} \\
& \left.-v_{(k 1)(12)(21)(k q)^{*}}+v_{(12)(k 1)(21)(k q)^{*}}\right)\end{aligned}$ \\
\hline$\Pi_{1}(2,1,-1) \otimes \Pi_{2}(1,1)$ & $\begin{aligned} \sum_{k} & \left(v_{(1 k)(22)(11)(p k)^{*}}-v_{(22)(1 k)(11)(p k)^{*}}\right. \\
& +v_{(11)(22)(1 k)(p k)^{*}}-v_{(22)(11)(1 k)(p k)^{*}} \\
& -v_{(1 k)(21)(12)(p k)^{*}}+v_{(21)(1 k)(12)(p k)^{*}} \\
& \left.-v_{(1 k)(12)(21)(p k)^{*}}+v_{(12)(1 k)(21)(p k)^{*}}\right)\end{aligned}$ \\
\hline$\Pi_{1}(2,1,-1) \otimes \Pi_{2}(2,1,-1)$ & $\begin{array}{l}v_{(11)(22)(11)(p q)^{*}}-v_{(22)(11)(11)(p q)^{*}} \\
\quad-v_{(11)(21)(12)(p q)^{*}}+v_{(21)(11)(12)(p q)^{*}} \\
\quad-v_{(11)(12)(21)(p q)^{*}}+v_{(12)(11)(21)(p q)^{*}}\end{array}$ \\
\hline
\end{tabular}

Table 2 . 
where $X, Y \in \mathcal{X}$, the algebra of $C^{\infty}$ vector fields on $M$.

Let $T_{m} M$ be the tangent space at $m$. Then, having in mind the above exposition we have $T_{m} M=V^{p} \otimes V^{q}$, where $V^{p}$ and $V^{q}$ are fibers of $E$ and $H$ respectively. We denote by $\mathcal{R}(M)$ the vector bundle with fiber $\mathcal{R}\left(T_{m} M\right)$, i.e. the vector space of curvature tensors at a point $m \in M$. Theorem 3.3 applied to $\mathcal{R}\left(T_{m} M\right)$ gives rise to a decomposition of $\mathcal{R}(M)$ into subbundles. We still denote the simple components as in the previous sections.

Let us recall that our results in Section 3 are pure algebraic. To obtain geometrical interpretations of the components (which are bundles in the setting of this section) in Theorem 3.3, we need to find some torsion-free connections whose curvature tensors belong to these components.

Many authors have been interested in this topic giving previously partially geometrical interpretations of some components or their direct sum. We mention here some of previously known examples and find some new ones.

Under appropriate assumptions, for example, when the Grassmann structure is associated with a quaternionic Kähler structure on $M$, halfflatness implies the Yang-Mills equations. Inspired by the harmonic space approach, Alekseevsky, Cortés and Devchand [3] have developed a local construction of (holomorphic) half-flat connections $D^{W}$ over a complex manifold with (holomorphic) Grassmann structure equipped with a suitable linear connection, in general case with torsion. Any such connection $D^{W}$ can be obtained from a prepotential by solving a system of linear first order ODEs.

Machida and Sato [34] have regarded a Grassmann structure of type $(p, q)$ as a geometric structure related to a simple graded Lie algebra of the first kind. They have applied the Tanaka theory which induces the existence of a unique normal Cartan connection and explicitly have written down the normal Cartan connection for a Grassmann structure of type $(p, q)$.

\subsection{Curvatures of half-flat torsion-free connections}

Among Grassmann connections we study mainly half-flat ones. Let $M$ be a manifold with a Grassmann structure $T M \simeq E \otimes H$ of type $(p, q)$ and with a Grassmann connection $D=D^{E} \otimes I d+I d \otimes D^{H}$. Then $D$ is called positive half-flat if the connection $D^{H}$ in the vector bundle $H \rightarrow M$ is flat and negative half-flat if $D^{E}$ is flat in the vector bundle $E \rightarrow M$. The corresponding structures $T M \simeq E \otimes H$ are called a positive half-flat Grassmann structure and a negative half-flat Grassmann structure respec- 
tively. In general case $D$ is not torsion-free, but we are interested only in the connections which are torsion-free. We call them half-flat torsion-free connections and the corresponding Grassmann structures half-flat torsionfree structures. The Levi-Civita connection on a hyper-Kähler manifold is an example of a positive half-flat torsion-free connection [3]. The conditions for $D$ being torsion-free have been studied in [34, Theorems 5.1, 6.1, 7.3].

We are interested now in curvatures for half-flat torsion-free Grassmann connections. Let us recall that $\mathcal{R}(M)$ is the vector bundle with fiber $\mathcal{R}\left(T_{m} M\right)$ - the vector space of curvature tensors at a point $m \in M$. The tensor spaces and the tensor fields on $M$ give rise to decompositions with respect to the same group $G$.

Denote by $\Lambda^{2}$ the space of 2 -forms $\Lambda^{2}(T M)$ or $\Lambda^{2}\left(T^{*} M\right)$. Then $\Lambda^{2}$ is decomposed (see Lemma $3.2(i 2)$ ) as follows

$$
\Lambda^{2}=S^{2}(E) \otimes \Lambda^{2}(H) \oplus \Lambda^{2}(E) \otimes S^{2}(H)
$$

The decomposition is invariant under the group $G$. We combine (15) with our restriction (the vanishing of torsion is equivalent to the first Bianchi identity) to get

$$
\mathcal{R}(M)=\left\{R \in \Lambda^{2} \otimes \mathfrak{g} \mid \sigma_{c y} R(x, y) z=0\right\}
$$

whose subbundles

$$
\begin{aligned}
& \mathcal{R}^{+}(M)=\left\{R \in S^{2}(E) \otimes \Lambda^{2}(H) \otimes \mathfrak{g} \mid \sigma_{c y} R(x, y) z=0\right\}, \\
& \mathcal{R}^{-}(M)=\left\{R \in \Lambda^{2}(E) \otimes S^{2}(H) \otimes \mathfrak{g} \mid \sigma_{c y} R(x, y) z=0\right\},
\end{aligned}
$$

consist of curvatures for positive and negative torsion-free half-flat Grassmann connections respectively (see [3] and [34]).

We combine now (17) with Theorem 3.3 to get

Theorem 5.1. Let $G=G L(p) \otimes G L(q)$ and let $\mathcal{R}^{+}(M)$ be the subbundle that consists of curvatures of a manifold with a positive torsion-free half-flat Grassmann structure. Then $\mathcal{R}^{+}(M)$ decomposes $(p, q>3)$ as follows

$$
\begin{aligned}
\mathcal{R}^{+}(M)=\left(\Pi_{1}^{t s}(2) \oplus \Pi_{1}(3,-1)\right) \otimes \mathcal{R}\left(V^{q}\right) \\
\oplus \mathcal{R}^{*}\left(V^{p}\right) \otimes\left(\Pi_{2}^{t a}(1,1) \oplus \Pi_{2}(1,1,1,-1)\right) .
\end{aligned}
$$


Proof. It is clear that all components in the righthand side of (19) belong to $\mathcal{R}^{+}(M)$. Other candidates in Theorem 3.3 should be omitted either because of skew-symmetry condition or because of the Bianchi identity, since $\mathcal{R}(M)=\mathcal{R}^{+}(M) \oplus \mathcal{R}^{-}(M) \oplus \tilde{\mathcal{R}}(M)$, where $\tilde{\mathcal{R}}(M)=\left[\Pi_{1}(2,1) \otimes\right.$ $\left.\Pi_{2}(2,1)\right] \otimes \Pi_{1}(-1) \otimes \Pi_{2}(-1)$. See (12) for more details.

A similar statement holds for $\mathcal{R}^{-}(M)$.

Let us emphasize that $\tilde{\mathcal{R}}(M)$ consists of curvatures that fulfill the condition (6) but are not obtained by combining the symmetries with respect to $E$ and $H$. See also $P_{\Pi_{1}(2) \otimes \Pi_{2}(2)} R$ in Proposition 5.14.

Consequently, one can obtain algebraic obstructions for a torsion-free connection defined on $M(T M \simeq E \otimes H)$ being positive half-flat torsion-free.

COROLlaRY 5.2. Let $D$ be a torsion-free non-flat connection defined globally (or locally) on $M$ with a Grassmann structure of type $(p, q)$. Then $D$ is not positive half-flat iff its curvature tensor has non-vanishing projection on the subbundle

$$
\begin{aligned}
\mathcal{R}^{-} & (M) \oplus \Pi_{1}(2) \otimes \Pi_{2}(2) \oplus \Pi_{1}(1,1) \otimes \Pi_{2}(1,1) \oplus \Pi_{1}(2) \otimes \Pi_{2}(1,1) \\
& \oplus \Pi_{1}(1,1) \otimes \Pi_{2}(2) \oplus \Pi_{1}(2,1,-1) \otimes \Pi_{2}(2) \oplus \Pi_{1}(2) \otimes \Pi_{2}(2,1,-1) \\
& \oplus \Pi_{1}(2,1,-1) \otimes \Pi_{2}(1,1) \oplus \Pi_{1}(1,1) \otimes \Pi_{2}(2,1,-1) \\
& \oplus \Pi_{1}(2,1,-1) \otimes \Pi_{2}(2,1,-1) .
\end{aligned}
$$

Proof. Use Theorem 3.3, Theorem 5.1 and Definition of half-flat torsion-free connections.

Analogous statement holds for negative half-flat torsion-free-connection.

\subsection{Projective invariants}

The main purpose of this subsection is to obtain some invariants of projective transformations of real Grassmann manifolds and to analyze them in the light of Theorem 3.3. Therefore, in what follows, we give some results concerning torsion-free connections $D$ and $D^{\prime}$ on $\mathcal{G} r\left(V^{p \otimes q}\right)$ with common geodesic lines. With this aim, we introduce the $(1,2)$-rank tensor field $T$ whose components $T_{(i \alpha)(j \beta)}^{(k \gamma)}$ are given by

$$
T_{(i \alpha)(j \beta)}^{(k \gamma)}:=\Gamma_{(i \alpha)(j \beta)}^{(k \gamma)}-G_{(i \alpha)(j \beta)}^{(k \gamma)},
$$


where $\Gamma_{(i \alpha)(j \beta)}^{(k \gamma)}$ and $G_{(i \alpha)(j \beta)}^{(k \gamma)}$ denote the Christoffel symbols of $D$ and $D^{\prime}$ respectively and $i, j, k, \ldots \in\{1, \ldots, p\}, \alpha, \beta, \gamma \in\{1, \ldots, q\}$. Now we can state

Lemma 5.3. Let $\mathcal{G} r\left(V^{p \otimes q}\right)$ be a Grassmann manifold, $D, D^{\prime}$ two torsion-free connections on $\mathcal{G} r\left(V^{p \otimes q}\right)$ with corresponding Christoffel symbols $\Gamma_{(i \alpha)(j \beta)}^{(k \gamma)}$ and $G_{(i \alpha)(j \beta)}^{(k \gamma)}$ respectively. Then these connections are projectively related if and only if their Christoffel symbols satisfy the equation

$$
G_{(i \alpha)(j \beta)}^{(k \gamma)}=\Gamma_{(i \alpha)(j \beta)}^{(k \gamma)}-\left(\eta_{(i \alpha)} \delta_{j}^{k} \delta_{\beta}^{\gamma}+\eta_{(j \beta)} \delta_{i}^{k} \delta_{\alpha}^{\gamma}\right)
$$

where

$$
\eta_{(i \alpha)}=\frac{1}{p q+1} T_{(k \gamma)(i \alpha)}^{(k \gamma)}
$$

Proof. See [23].

Let now $\mathcal{B}=\left\{z_{l \tau}=x_{l} \otimes y_{\tau} \mid 1 \leq l \leq p, 1 \leq \tau \leq q\right\}$ be a fixed basis of the tangent space $T_{m}\left(\mathcal{G} r\left(V^{p \otimes q}\right)\right)=V^{p} \otimes V^{q}$ and let $D$ be a torsion-free connection on $\mathcal{G} r\left(V^{p \otimes q}\right)$ with Christoffel symbols $\Gamma_{(i \alpha)(j \beta)}^{(k \gamma)}$. Then one can consider its curvature tensor

$$
R\left(z_{i \alpha}, z_{j \beta}\right) z_{k \gamma}=D_{z_{i \alpha}} D_{z_{j \beta}} z_{k \gamma}-D_{z_{j \beta}} D_{z_{i \alpha}} z_{k \gamma}-D_{\left[z_{i \alpha}, z_{j \beta}\right]} z_{k \gamma}
$$

with components

$$
\begin{aligned}
R_{(i \alpha)(j \beta)(k \gamma)}^{(l \nu)}= & \frac{\partial \Gamma_{(j \beta)(k \gamma)}^{(l \nu)}}{\partial x^{i \alpha}}-\frac{\partial \Gamma_{(i \alpha)(k \gamma)}^{(l \nu)}}{\partial x^{j \beta}} \\
& \quad+\Gamma_{(i \alpha)(k \gamma)}^{(m \mu)} \Gamma_{(j \beta)(m \mu)}^{(l \nu)}-\Gamma_{(j \beta)(k \gamma)}^{(m \mu)} \Gamma_{(i \alpha)(m \mu)}^{(l \nu)},
\end{aligned}
$$

and the Ricci trace $\rho_{R}^{11}=\operatorname{con}((1,1)(4,4)) R$. In particular, if we suppose that $R$ and $R^{\prime}$ are the curvature tensor fields of the projectively related torsion-free connections $D$ and $D^{\prime}$ respectively, then, from Lemma 5.3, we get that their Ricci traces $\rho_{R}^{11}$ and $\rho_{R^{\prime}}^{11}$ satisfy the following equation

$$
\rho_{R^{\prime}}^{11}\left(z_{i \alpha}, z_{j \beta}\right)=\rho_{R}^{11}\left(z_{i \alpha}, z_{j \beta}\right)+p q \eta\left(z_{i \alpha}, z_{j \beta}\right)-\eta\left(z_{j \beta}, z_{i \alpha}\right)
$$

where

$$
\eta_{(i \alpha)(j \beta)}=\eta\left(z_{i \alpha}, z_{j \beta}\right):=-\left(D_{z_{i \alpha}} \eta\right)\left(z_{j \beta}\right)+\eta\left(z_{i \alpha}\right) \eta\left(z_{j \beta}\right) .
$$

Analogously to the well known definitions, (see also [22], [23], [24]) we can now introduce the following. 
Definition 5.4. Let $D$ be a torsion-free connection on the real Grassmann manifold $\mathcal{G} r\left(V^{p \otimes q}\right)$ and let $R, \rho_{R}^{11}$ be its curvature tensor field and its Ricci curvature. Then the (1,3)-tensor field given by

$$
\begin{gathered}
P\left(z_{i \alpha}, z_{j \beta}\right) z_{k \gamma}=R\left(z_{i \alpha}, z_{j \beta}\right) z_{k \gamma}-\left(Q\left(z_{i \alpha}, z_{j \beta}\right)-Q\left(z_{j \beta}, z_{i \alpha}\right)\right) z_{k \gamma} \\
+Q\left(z_{j \beta}, z_{k \gamma}\right) z_{i \alpha}-Q\left(z_{i \alpha}, z_{k \gamma}\right) z_{j \beta}
\end{gathered}
$$

where

$$
Q\left(z_{i \alpha}, z_{j \beta}\right)=-\frac{p q}{p^{2} q^{2}-1} \rho_{R}^{11}\left(z_{i \alpha}, z_{j \beta}\right)-\frac{1}{p^{2} q^{2}-1} \rho_{R}^{11}\left(z_{j \beta}, z_{i \alpha}\right),
$$

is the projective curvature tensor associated with $D$.

In fact, taking into account that (25) implies the following transformation formula for $Q$ :

$$
Q^{\prime}\left(z_{i \alpha}, z_{j \beta}\right)=Q\left(z_{i \alpha}, z_{j \beta}\right)-\eta\left(z_{i \alpha}, z_{j \beta}\right),
$$

from Lemma 5.3 we get that the tensor $P$ is invariant for projective transformations of the connection $D$. Moreover, its definition assures that $P$ is a curvature tensor satisfying the symmetries (6) and (7) as well as that its Ricci trace $\rho_{P}^{11}$ vanishes. For more details one can see [23].

Let $\mathcal{P}\left(\mathcal{G} r\left(V^{p \otimes q}\right)\right) \subseteq \mathcal{R}\left(\mathcal{G} r\left(V^{p \otimes q}\right)\right)$ be the vector subbundle of projective curvature tensors. Consequently, comparing the properties of $\mathcal{P}\left(\mathcal{G} r\left(V^{p \otimes q}\right)\right)$ with the decomposition formula in Theorem 3.3, we obtain that $\mathcal{P}\left(\mathcal{G} r\left(V^{p \otimes q}\right)\right)$ is not simple. More precisely we state the following theorem.

TheOREM 5.5. The decomposition of subbundle $\mathcal{P}\left(\mathcal{G} r\left(V^{p \otimes q}\right)\right)$ of $\mathcal{R}\left(\mathcal{G} r\left(V^{p \otimes q}\right)\right)$ into direct sum of simple $G$-subbundles is given by $(p, q>3)$

$$
\begin{aligned}
\mathcal{P}\left(\mathcal{G} r\left(V^{p \otimes q}\right)\right)= & 2 \Pi_{1}(2) \otimes \Pi_{2}(2) \oplus 2 \Pi_{1}(1,1) \otimes \Pi_{2}(1,1) \\
& \oplus 2 \Pi_{1}(2) \otimes \Pi_{2}(1,1) \oplus 2 \Pi_{1}(1,1) \otimes \Pi_{2}(2) \\
& \oplus \Pi_{1}^{t s}(2) \otimes \Pi_{2}(2,1,-1) \oplus \Pi_{1}(2,1,-1) \otimes \Pi_{2}^{t s}(2) \\
& \oplus \Pi_{1}(3,-1) \otimes \mathcal{R}\left(V^{q}\right) \oplus \mathcal{R}\left(V^{p}\right) \otimes \Pi_{2}(3,-1) \\
& \oplus \Pi_{1}(1,1,1,-1) \otimes \mathcal{R}^{*}\left(V^{q}\right) \oplus \mathcal{R}^{*}\left(V^{p}\right) \otimes \Pi_{2}(1,1,1,-1) \\
& \oplus \Pi_{1}^{t a}(1,1) \otimes \Pi_{2}(2,1,-1) \oplus \Pi_{1}(2,1,-1) \otimes \Pi_{2}^{t a}(1,1) \\
& \oplus \Pi_{1}(2,1,-1) \otimes \Pi_{2}(2) \oplus \Pi_{1}(2) \otimes \Pi_{2}(2,1,-1) \\
& \oplus \Pi_{1}(2,1,-1) \otimes \Pi_{2}(1,1) \oplus \Pi_{1}(1,1) \otimes \Pi_{2}(2,1,-1) \\
& \oplus \Pi_{1}(2,1,-1) \otimes \Pi_{2}(2,1,-1) .
\end{aligned}
$$


Remark 5.6. Th. Hangan in [22] has also studied the projective transformations of $\mathcal{G} r\left(V^{p \otimes q}\right)$ from pseudogroups point of view.

We are now interested in projection operators on some components considered in Theorem 3.3. To obtain these projections into some of simple components we introduce the following notations:

$$
\begin{aligned}
& \mathcal{S}_{(l \mu)(j \beta)}=\frac{1}{2}\left(\rho_{(l \mu)(j \beta)}^{11}+\rho_{(j \beta)(l \mu)}^{11}\right), \\
& \mathcal{A}_{(l \mu)(j \beta)}= \frac{1}{2}\left(\rho_{(l \mu)(j \beta)}^{11}-\rho_{(j \beta)(l \mu)}^{11}\right), \\
& \mathcal{S}_{(l \mu)(j \beta)}^{s}=\frac{1}{4}\left[\rho_{(j \beta)(l \mu)}^{11}+\rho_{(l \mu)(j \beta)}^{11}+\rho_{(l \beta)(j \mu)}^{11}+\rho_{(j \mu)(l \beta)}^{11}\right], \\
& \mathcal{S}_{(l \mu)(j \beta)}^{a}=\frac{1}{4}\left[\rho_{(j \beta)(l \mu)}^{11}+\rho_{(l \mu)(j \beta)}^{11}-\rho_{(l \beta)(j \mu)}^{11}-\rho_{(j \mu)(l \beta)}^{11}\right], \\
& \mathcal{A}_{(l \mu)(j \beta)}^{s}=\frac{1}{4}\left[\rho_{(j \beta)(l \mu)}^{11}+\rho_{(l \beta)(j \mu)}^{11}-\rho_{(l \mu)(j \beta)}^{11}-\rho_{(j \mu)(l \beta)}^{11}\right], \\
& \mathcal{A}_{(l \mu)(j \beta)}^{a}=\frac{1}{4}\left[\rho_{(j \beta)(l \mu)}^{11}-\rho_{(l \beta)(j \mu)}^{11}-\rho_{(l \mu)(j \beta)}^{11}+\rho_{(j \mu)(l \beta)}^{11}\right] .
\end{aligned}
$$

Consequently, we use our previous computations to find:

$$
\begin{aligned}
\left(P_{\Pi(2) \otimes \Pi(2)} R\right)_{(l \mu)(j \beta)(k \gamma)}{ }^{(i \alpha)} & =\frac{1}{4(p q-1)}\left[\delta_{\mu}^{\alpha} \delta_{l}^{i} \mathcal{S}_{(j \beta)(k \gamma)}^{s}-\delta_{\beta}^{\alpha} \delta_{j}^{i} \mathcal{S}_{(l \mu)(k \gamma)}^{s}\right] \\
\left(P_{\Pi(1,1) \otimes \Pi(1,1)} R\right)_{(l \mu)(j \beta)(k \gamma)}{ }^{(i \alpha)} & =\frac{1}{4(p q-1)}\left[\delta_{\mu}^{\alpha} \delta_{l}^{i} \mathcal{S}_{(k \gamma)(j \beta)}^{a}-\delta_{\beta}^{\alpha} \delta_{j}^{i} \mathcal{S}_{(k \gamma)(l \mu)}^{a}\right] \\
\left(P_{\Pi(2) \otimes \Pi(1,1)} R\right)_{(l \mu)(j \beta)(k \gamma)}{ }^{(i \alpha)} & =\frac{1}{4(p q+1)}\left[\delta_{\mu}^{\alpha} \delta_{l}^{i} \mathcal{A}_{(j \beta)(k \gamma)}^{s}\right. \\
\left(P_{\Pi(1,1) \otimes \Pi(2)} R\right)_{(l \mu)(j \beta)(k \gamma)}{ }^{(i \alpha)}= & \frac{1}{4(p q+1)}\left[\delta_{\beta}^{\alpha} \delta_{j}^{i} \mathcal{A}_{(l \mu)(k \gamma)}^{s} \delta_{l}^{i} \mathcal{A}_{(j \beta)(k \gamma)}^{a} \delta_{\gamma}^{\alpha} \delta_{k}^{i} \mathcal{A}_{(l \mu)(j \beta)}^{s}\right] \\
& \left.-\delta_{\beta}^{\alpha} \delta_{j}^{i} \mathcal{A}_{(l \mu)(k \gamma)}^{a}+2 \delta_{\gamma}^{\alpha} \delta_{k}^{i} \mathcal{A}_{(j \beta)(l \mu)}^{a}\right]
\end{aligned}
$$

We prefer to omit other projections as they are rather complicated. We remark that they are determined by the corresponding Young symmetrizers, given above in Section 3. Anyhow we recall that

$$
\begin{aligned}
P_{\pi(2,1,-1)}=I d & -P_{\Pi_{1}(2) \otimes \Pi_{2}(2)}-P_{\Pi_{1}(1,1) \otimes \Pi_{2}(1,1)} \\
& -P_{\Pi_{1}(2) \otimes \Pi_{2}(1,1)}-P_{\Pi_{1}(1,1) \otimes \Pi_{2}(2)}
\end{aligned}
$$


is the projector on subbundle $\mathcal{P}\left(\mathcal{G} r\left(V^{p \otimes q}\right)\right)$, invariant under projective transformations assuming that the corresponding projections in this setting are given by (29). We emphasize that these projection operators are uniquely determined if we wish $P_{\pi(2,1,-1)} R$, given by $(30)$, to be projectively invariant.

We also give here the projections of the curvature for the Leichtweiss metric [32] in order to point out its projectively invariant components. We consider this curvature in Subsection 5.4 too, to obtain other type of projection operators to show that they are not uniquely determined and that corresponding components may have various interpretations.

Proposition 5.7. On the Grassmann manifold $\mathcal{G} r\left(V^{p \otimes q}\right), p q>1$, there exists a Riemannian metric

$$
d s^{2}=\lambda\left(\sum_{i=1}^{p} \sum_{\alpha=p+1}^{p+q}\left(\left\langle e_{\alpha} d e_{i}\right\rangle\right)^{2}\right),
$$

where $\lambda=$ const $>0$, such that $\mathcal{G} r\left(V^{p \otimes q}\right)$ is an Einstein space and the Riemann curvature tensor $R$ satisfies the relations

(i1) $P_{\pi(2)} R=P_{\Pi_{1}(2) \otimes \Pi_{2}(2)} R, \quad P_{\pi(1,1)} R=0$,

(i2) $P_{\pi(2,1,-1)} R=P_{\Pi_{1}(2) \otimes \Pi_{2}(2)}^{\prime} R \oplus P_{\Pi_{1}(2) \otimes \Pi_{2}(2)}^{\prime \prime} R$, for $p \neq q$, and

(i3) $P_{\pi(2,1,-1)} R=P_{\Pi_{1}(2) \otimes \Pi_{2}(2)}^{\prime} R$, for $p=q$.

Moreover:

$$
\begin{gathered}
\left(P_{\pi(2)} R\right)_{(i \alpha)(j \beta)(k \gamma)}{ }^{(l \nu)}=\frac{2-p-q}{1-p q}\left(\delta_{j k} \delta_{i}^{l} \delta_{\beta \gamma} \delta_{\alpha}^{\nu}-\delta_{i k} \delta_{j}^{l} \delta_{\alpha \gamma} \delta_{\beta}^{\nu}\right) \\
\left(P_{\pi(2,1,-1)} R\right)_{(i \alpha)(j \beta)(k \gamma)}{ }^{(l \nu)}=W_{(i \alpha)(j \beta)(k \gamma)}{ }^{(l \nu)} \\
=\left(\delta_{i k} \delta_{j}^{l}-\delta_{j k} \delta_{i}^{l}\right) \delta_{\alpha \beta} \delta_{\gamma}^{\nu}+\delta_{i j} \delta_{k}^{l}\left(\delta_{\alpha \gamma} \delta_{\beta}^{\nu}-\delta_{\beta \gamma} \delta_{\alpha}^{\nu}\right) \\
+\frac{p+q-2}{1-p q}\left(\delta_{j k} \delta_{i}^{l} \delta_{\beta \gamma} \delta_{\alpha}^{\nu}-\delta_{i k} \delta_{j}^{l} \delta_{\alpha \gamma} \delta_{\beta}^{\nu}\right)
\end{gathered}
$$

Proof. One can use [32] (see also Subsection 5.4) to see that the curvature tensor of the Leichtweiss metric in the origin of a coordinate system is given by the following relations

$$
\begin{aligned}
R_{(i \alpha)(j \beta)(k \gamma)}{ }^{(l \nu)}(0, \ldots, 0)=( & \left.\delta_{i k} \delta_{j}^{l}-\delta_{j k} \delta_{i}^{l}\right) \delta_{\alpha \beta} \delta_{\gamma}^{\nu} \\
& +\delta_{i j} \delta_{k}^{l}\left(\delta_{\alpha \gamma} \delta_{\beta}^{\nu}-\delta_{\beta \gamma} \delta_{\alpha}^{\nu}\right)
\end{aligned}
$$


Its Ricci traces are as follows

$$
\begin{aligned}
\left(\rho^{11}\right)_{(j \beta)(k \gamma)}(0, \ldots, 0) & =(2-p-q) \delta_{j k} \delta_{\beta \gamma} \\
& =(2-p-q) g_{(j \beta)(k \gamma)}(0, \ldots, 0), \\
\left(\rho^{12}\right)_{(j \beta)(k \gamma)}(0, \ldots, 0) & =(p-q) \delta_{j k} \delta_{\beta \gamma} \\
& =(p-q) g_{(j \beta)(k \gamma)}(0, \ldots, 0), \\
\left(\rho^{13}\right)_{(j \beta)(k \gamma)}(0, \ldots, 0) & =q(p-1) \delta_{j k} \delta_{\beta \gamma} \\
& =q(p-1) g_{(j \beta)(k \gamma)}(0, \ldots, 0) .
\end{aligned}
$$

Consequently we get our statements by using Theorems 5.5, 2.1.

\subsection{Normalization of Grassmann manifold}

As it is well known a manifold $M$ admits a torsion-free connection $D$ with symmetric Ricci tensor $\rho^{D}$ if and only if $M$ admits a $D$-parallel volume form $\omega=\omega(D)$, i.e. $D \omega=0$. The form $\omega$ is unique modulo a constant nonzero factor. But in the framework of the Grassmann manifolds $\mathcal{G} r\left(V^{p \otimes q}\right)$ there exists a distinguished subclass of torsion-free connections, that does not exist otherwise. They are obtained by means of certain constructions called normalizations, which we are now going to describe. The Grassmann manifold $\mathcal{G} r\left(V^{p \otimes q}\right)$ is said to be normalized if to each of its $p$-dimensional subspaces $U$ there corresponds a chosen subspace $U^{*}$ of dimension $q$ in the space $\mathbb{R}^{p+q}$, such that $U^{*}$ is a complement of $U$ in $\mathbb{R}^{p+q}$. The $U^{*}$ is called the normalizing subspace for $U$, while the Grassmann manifold $\mathcal{G} r\left(V^{p \otimes q}\right)$ is called normalized and denoted by $\mathcal{G} r^{\nu}\left(V^{p \otimes q}\right)$. We refer to [1] for more details.

A normalization of the Grassmann manifold $\mathcal{G} r\left(V^{p \otimes q}\right)$ is defined by a differential mapping $\nu: \mathcal{G} r\left(V^{p} \otimes V^{q}\right) \rightarrow \mathcal{G} r\left(V^{q} \otimes V^{p}\right)$ whose differential equations are the following

$$
\omega_{(i \alpha)}=\lambda_{(i \alpha)(j \beta)} \omega_{(j \beta)},
$$

where the 1-forms $\omega^{i \alpha}$ are basis forms of the frame bundle associated with $\mathcal{G} r\left(V^{p \otimes q}\right)$ and the coefficients $\lambda_{(i \alpha)(j \beta)}$ form a tensor called the fundamental tensor of $\mathcal{G} r^{\nu}\left(V^{p \otimes q}\right)$. The normalization $\nu$ is harmonic if the coefficients in equation (37) are symmetric with respect to the pair of indices $(j \beta),(i \alpha)$, i.e., $\lambda_{(i \alpha)(j \beta)}=\lambda_{(j \beta)(i \alpha)}$. When the fundamental tensor $\lambda_{(i \alpha)(j \beta)}$ satisfies the relation

$$
\lambda_{(i \alpha)(j \beta)}=-g_{i j} g_{\alpha \beta},
$$


with $g_{i j}, g_{\alpha \beta}$ symmetric, then the corresponding normalization $\nu$ is said to be polar and the normalized Grassmann manifold is homogeneous. It is clear that a polar normalization is also harmonic.

THEOREM 5.8. ([1]) The normalization $\nu$ of a normalized domain $U^{\nu} \subseteq$ $\mathcal{G} r\left(V^{p \otimes q}\right)$ uniquely determines a torsion-free affine connection $D^{\nu}$ with the connection forms

$$
\omega_{(i \alpha)(j \beta)}=\delta_{\alpha \beta} \omega_{i j}-\delta_{i j} \omega_{\alpha \beta},
$$

where $\omega_{\alpha \beta}$ and $\omega_{i j}$ are fiber forms of the frame bundle associated with a normalized Grassmann manifold $\mathcal{G} r^{\nu}\left(V^{p \otimes q}\right)$ (or a domain $U^{\nu}$ of this manifold).

One can use Theorem 5.8 to see which projections of the curvature corresponding to a connection $D^{\nu}$ vanish. The curvature tensor of this connection is expressed in terms of the fundamental tensor of the normalization $\nu$ according to the formulas

$$
\begin{aligned}
R_{(k \gamma)(l \mu)(j \beta)}^{(i \alpha)}=\frac{1}{2} & \left(\delta_{\beta}^{\alpha} \delta_{k}^{i} \lambda_{(j \gamma)(l \mu)}+\delta_{\gamma}^{\alpha} \delta_{j}^{i} \lambda_{(k \beta)(l \mu)}\right. \\
& \left.-\delta_{\beta}^{\alpha} \delta_{l}^{i} \lambda_{(j \mu)(k \gamma)}-\delta_{\mu}^{\alpha} \delta_{j}^{i} \lambda_{(l \beta)(k \gamma)}\right)
\end{aligned}
$$

To find the Ricci traces $\rho^{1 i}$ of the connection $D^{\nu}$ we need to contract the tensor (39) with respect to the corresponding indices. Consequently we get

$$
\begin{aligned}
\rho_{(l \mu)(j \beta)}^{11} & =R_{(i \alpha)(l \mu)(j \beta)}{ }^{(i \alpha)} \\
& =\frac{1}{2}\left((p+q) \lambda_{(j \beta)(l \mu)}-\lambda_{(j \mu)(l \beta)}-\lambda_{(l \beta)(j \mu)}\right), \\
\rho_{(l \gamma)(j \beta)}^{12} & =R_{(i \gamma)(l \alpha)(j \beta)}{ }^{(i \alpha)}=\frac{1}{2}\left(p \lambda_{(j \gamma)(l \beta)}-q \lambda_{(l \beta)(j \gamma)}\right), \\
\rho_{(l \gamma)(j \mu)}^{13} & =R_{(i \gamma)(l \mu)(j \alpha)}{ }^{(i \alpha)} \\
& =\frac{1}{2}\left((p q+1) \lambda_{(j \gamma)(l \mu)}-q \lambda_{(j \mu)(l \gamma)}-\lambda_{(l \mu)(j \gamma)}\right) .
\end{aligned}
$$

From these relations it follows immediately that the Ricci tensor $\rho^{11}$ of the connection $D^{\nu}$ is symmetric if and only if the normalization $\nu$ of the Grassmann manifold $\mathcal{G} r\left(V^{p \otimes q}\right)$ is harmonic.

Having in mind the results mentioned above, we can reach the geometrical interpretations of some components in our complete decomposition of $\mathcal{R}\left(\mathcal{G} r\left(V^{p \otimes q}\right)\right)$. We now show that we can characterize the curvature tensor 
at any point of $\mathcal{G} r\left(V^{p \otimes q}\right)$ corresponding to connections $D^{\nu}$ for an arbitrary normalization, as well as some subspaces related to the connections $D^{\nu}$ for special types of normalizations. To obtain a characterization of the components in a neighborhood we need the existence of local coordinate system where the curvature tensor of $D^{\nu}$ has just these components. We illustrate these ones by some examples in Subsections 5.4 and 5.6. Besides that, we give also the projection operators. Our decomposition of $\mathcal{R}\left(\mathcal{G} r\left(V^{p \otimes q}\right)\right)$ inform us what are the obstructions for their elements being a curvature tensor corresponding to some torsion-free connection that admits a normalization. While these obstructions are both of global and local nature, to have an affirmative answer of the first question it is not enough to use only our complete decomposition but also some topological tools, that is a topic of our forthcoming paper.

Proposition 5.9. Let $R\left(D^{\nu}\right) \in \mathcal{R}\left(\mathcal{G} r\left(V^{p \otimes q}\right)\right)$ be a curvature tensor of torsion-free connection $D^{\nu}$ corresponding to the normalization $\nu$ on $\mathcal{G} r\left(V^{p \otimes q}\right)$. Then it satisfies

$$
\begin{array}{r}
R\left(D^{\nu}\right) \in \Pi_{1}(2) \otimes \Pi_{2}(2) \oplus \Pi_{1}(1,1) \otimes \Pi_{2}(1,1) \\
\oplus \Pi_{1}(2) \otimes \Pi_{2}(1,1) \oplus \Pi_{1}(1,1) \otimes \Pi_{2}(2),
\end{array}
$$

with corresponding projections as follows:

$$
\begin{aligned}
& \left(P_{\Pi(2) \otimes \Pi(2)} R\right)_{(k \gamma)(l \mu)(j \beta)}{ }^{(i \alpha)}=\frac{1}{2}\left[\delta_{\beta}^{\alpha}\left(\delta_{k}^{i} S_{(j \gamma)(l \mu)}^{s}-\delta_{l}^{i} S_{(j \gamma)(k \mu)}^{s}\right)\right. \\
& \left.+\delta_{j}^{i}\left(\delta_{\gamma}^{\alpha} S_{(k \beta)(l \mu)}^{s}-\delta_{\mu}^{\alpha} S_{(l \gamma)(k \beta)}^{s}\right)\right], \\
& \left(P_{\Pi(1,1) \otimes \Pi(1,1)} R\right)_{(k \gamma)(l \mu)(j \beta)}{ }^{(i \alpha)}=\frac{1}{2}\left[\delta_{\beta}^{\alpha}\left(\delta_{k}^{i} S_{(j \gamma)(l \mu)}^{a}+\delta_{l}^{i} S_{(j \gamma)(l \mu)}^{a}\right)\right. \\
& \left.+\delta_{j}^{i}\left(\delta_{\gamma}^{\alpha} S_{(k \beta)(l \mu)}^{a}+\delta_{\mu}^{\alpha} S_{(l \gamma)(k \beta)}^{a}\right)\right], \\
& \left(P_{\Pi(2) \otimes \Pi(1,1)} R\right)_{(k \gamma)(l \mu)(j \beta)}{ }^{(i \alpha)}=\frac{1}{2}\left[\delta_{\beta}^{\alpha}\left(\delta_{k}^{i} A_{(j \gamma)(l \mu)}^{s}+\delta_{l}^{i} A_{(j \gamma)(l \mu)}^{s}\right)\right. \\
& \left.+\delta_{j}^{i}\left(\delta_{\gamma}^{\alpha} A_{(k \beta)(l \mu)}^{s}+\delta_{\mu}^{\alpha} S_{(l \gamma)(k \beta)}^{s}\right)\right], \\
& \left(P_{\Pi(1,1) \otimes \Pi(2)} R\right)_{(k \gamma)(l \mu)(j \beta)}{ }^{(i \alpha)}=\frac{1}{2}\left[\delta_{\beta}^{\alpha}\left(\delta_{k}^{i} A_{(j \gamma)(l \mu)}^{a}-\delta_{l}^{i} A_{(j \gamma)(l \mu)}^{a}\right)\right. \\
& \left.+\delta_{j}^{i}\left(\delta_{\gamma}^{\alpha} A_{(k \beta)(l \mu)}^{a}-\delta_{\mu}^{\alpha} A_{(l \gamma)(k \beta)}^{a}\right)\right],
\end{aligned}
$$


where

$$
\begin{aligned}
S_{(l \mu)(j \beta)}^{s} & =\frac{1}{4}\left[\lambda_{(j \beta)(l \mu)}+\lambda_{(l \mu)(j \beta)}+\lambda_{(l \beta)(j \mu)}+\lambda_{(j \mu)(l \beta)}\right], \\
S_{(l \mu)(j \beta)}^{a} & =\frac{1}{4}\left[\lambda_{(j \beta)(l \mu)}+\lambda_{(l \mu)(j \beta)}-\lambda_{(l \beta)(j \mu)}-\lambda_{(j \mu)(l \beta)}\right], \\
A_{(l \mu)(j \beta)}^{s} & =\frac{1}{4}\left[\lambda_{(j \beta)(l \mu)}-\lambda_{(l \mu)(j \beta)}+\lambda_{(l \beta)(j \mu)}-\lambda_{(j \mu)(l \beta)}\right], \\
A_{(l \mu)(j \beta)}^{a} & =\frac{1}{4}\left[\lambda_{(j \beta)(l \mu)}-\lambda_{(l \mu)(j \beta)}-\lambda_{(l \beta)(j \mu)}+\lambda_{(j \mu)(l \beta)}\right] .
\end{aligned}
$$

Proof. We use Proposition 3.1 to see that in the general case the curvature tensor $R \in \mathcal{R}(V)$, corresponding to a torsion-free connection $D$, has three independent traces $\rho^{1 i}(i=1,2,3)$. But for a connection $D^{\nu}$ that permits a normalization $\nu$, because of (40)-(42), it follows that there exists only one independent trace whose components are linear combinations of $\lambda_{(j \gamma)(l \mu)}$. Therefore we combine Lemma 3.2 with these results to prove the first part of Proposition. The second part of Proposition one can check by using (39) and Lemma 3.2.

As we know, the normalized Grassmann manifold is not in general homogeneous (see [1, p. 260]). Then the polar normalization of a Grassmann manifold assures that it is homogeneous. Other important things (generally speaking at least) are the following: in this case $D^{\nu}$ is always the Levi-Civita connection of a metric on the polar-normalized manifold and in addition this manifold is always Einsteinian with respect to this metric.

COROLlary 5.10. Let $R\left(D^{h}\right) \in \mathcal{R}\left(\mathcal{G} r\left(V^{p \otimes q}\right)\right)$ be the curvature tensor of torsion-free connection $D^{h}$ corresponding to the harmonic normalization $h$ on $\mathcal{G} r\left(V^{p \otimes q}\right)$. Then

$$
R\left(D^{h}\right) \in \Pi_{1}(2) \otimes \Pi_{2}(2) \oplus \Pi_{1}(1,1) \otimes \Pi_{2}(1,1)
$$

with projections as in Proposition 5.9.

COROLlary 5.11. If a Grassmann manifold $\mathcal{G} r\left(V^{p \otimes q}\right)$ admits the polar normalization $\pi$ such that $\mathcal{G} r\left(V^{p \otimes q}\right)$ is homogeneous, then the corresponding connection $D^{\pi}$ satisfies the condition

$$
R\left(D^{\pi}\right) \in \Pi_{1}(2) \otimes \Pi_{2}(2)
$$


Let us introduce now the following subbundles of $\mathcal{R}\left(\mathcal{G} r\left(V^{p \otimes q}\right)\right)$

$$
\begin{gathered}
\mathcal{O}\left(\mathcal{G} r\left(V^{p \otimes q}\right)\right)=\Pi_{1}(3,-1) \otimes \mathcal{R}\left(V^{q}\right) \oplus \mathcal{R}\left(V^{p}\right) \otimes \Pi_{2}(3,-1) \\
\oplus \Pi_{1}(1,1,1,-1) \otimes \mathcal{R}^{*}\left(V^{q}\right) \oplus \mathcal{R}^{*}\left(V^{p}\right) \otimes \Pi_{2}(1,1,1,-1) \\
\oplus 2 \Pi_{1}(2,1,-1) \otimes \Pi_{2}(2) \oplus 2 \Pi_{1}(2) \otimes \Pi_{2}(2,1,-1) \\
\oplus 2 \Pi_{1}(2,1,-1) \otimes \Pi_{2}(1,1) \oplus 2 \Pi_{1}(1,1) \otimes \Pi_{2}(2,1,-1) \\
\oplus \Pi_{1}(2,1,-1) \otimes \Pi_{2}(2,1,-1), \\
\mathcal{H}\left(\mathcal{G} r\left(V^{p \otimes q}\right)\right)=\Pi_{1}(1,1) \otimes \Pi_{2}(2) \oplus \Pi_{1}(2) \otimes \Pi_{2}(1,1) \\
\oplus \Pi_{1}(1,1) \otimes \Pi_{2}(2) \oplus \Pi_{1}(1,1) \otimes \Pi_{2}(2) \\
\oplus \Pi_{1}(2) \otimes \Pi_{2}(1,1) \oplus \Pi_{1}(2) \otimes \Pi_{2}(1,1),
\end{gathered}
$$

and

$$
\begin{gathered}
\mathcal{P} o\left(\mathcal{G} r\left(V^{p \otimes q}\right)\right)=\Pi_{1}(1,1) \otimes \Pi_{2}(1,1) \oplus \Pi_{1}(1,1) \otimes \Pi_{2}(1,1) \\
\oplus \Pi_{1}(1,1) \otimes \Pi_{2}(1,1) .
\end{gathered}
$$

Proposition 5.12. Let $D$ be a torsion-free non-flat connection defined globally (or locally) on $\mathcal{G} r\left(V^{p \otimes q}\right)$. Then $D$ does not correspond to

(i1) any normalization if and only if its curvature tensor $R(D)$ has a nonvanishing projection on the subbundle $\mathcal{O}\left(\mathcal{G} r\left(V^{p \otimes q}\right)\right)$.

(i2) any harmonic normalization if and only if its curvature tensor $R(D)$ has a non-vanishing projection on the subbundle $\mathcal{O}\left(\mathcal{G} r\left(V^{p \otimes q}\right)\right) \oplus$ $\mathcal{H}\left(\mathcal{G} r\left(V^{p \otimes q}\right)\right)$.

(i3) any polar normalization such that $\mathcal{G} r\left(V^{p \otimes q}\right)$ is homogeneous if and only if its curvature tensor $R(D)$ has non-vanishing projection on the subbundle $\mathcal{O}\left(\mathcal{G} r\left(V^{p \otimes q}\right)\right) \oplus \mathcal{H}\left(\mathcal{G} r\left(V^{p \otimes q}\right)\right) \oplus \mathcal{P} o\left(\mathcal{G} r\left(V^{p \otimes q}\right)\right)$.

Proof. (i1) We use (39)-(42) to see that the torsion-free non-flat connection uniquely determined by a normalization of $\mathcal{G} r\left(V^{p \otimes q}\right)$ has all nonvanishing traces $\rho^{1 i}, i=1,2,3$ which are dependent on the fundamental tensor $\lambda$. Consequently, its curvature tensor may have non-vanishing projections only on the subbundles of type $\Pi_{1}(2) \otimes \Pi_{2}(2), \Pi_{1}(1,1) \otimes \Pi_{2}(1,1)$, $\Pi_{1}(2) \otimes \Pi_{2}(1,1), \Pi_{1}(1,1) \otimes \Pi_{2}(2)$. The converse part can be proved in a similar way.

( $i 2)$ Since the fundamental tensor $\lambda$ corresponding to a harmonic normalization $h$ is symmetric and vice versa, the proof follows directly from (i1).

For $(i 3)$ we use (38), the symmetry of $g_{i j}, g_{\alpha \beta}$ and $(i 1)$. 
Remark 5.13. Finally we notice that $D^{\nu}$ is flat if and only if $R\left(D^{\nu}\right)=0$ i.e. if and only if the domain $U^{\nu}$ is endowed with the structure of the affine space $A^{\rho}$ of dimension $\rho=p q$. We refer to [1] for more details.

As we have seen throughout this section various examples of torsion-free connections are already known on Grassmann manifolds $\mathcal{G} r\left(V^{p \otimes q}\right)$. Some new examples are given in the next subsections.

\subsection{Ricci symmetric connections}

For the first fundamental form

$$
d s^{2}=\sum_{i, j=1}^{p} \sum_{\alpha, \beta=p+1}^{p+q} g_{(i \alpha)(j \beta)}\left(x_{k \gamma}\right) d x_{i \alpha} d x_{j \beta}
$$

with

$$
g_{(i \alpha)(j \beta)}=\sum_{k=1}^{p} \sum_{\gamma=p+1}^{p+q}\left\langle e_{\gamma} \frac{\partial e_{k}}{\partial x_{i \alpha}}\right\rangle\left\langle e_{\gamma} \frac{\partial e_{k}}{\partial x_{j \beta}}\right\rangle
$$

we get the Pfaff forms $\omega_{(i \alpha)}$ using the vector differential forms

$$
d e_{i}=\sum_{j=1}^{p} \sum_{\beta=p+1}^{p+q} \frac{\partial e_{i}}{\partial x_{j \beta}} d x_{j \beta}, \quad d e_{\alpha}=\sum_{j=1}^{p} \sum_{\beta=p+1}^{p+q} \frac{\partial e_{\alpha}}{\partial x_{j \beta}} d x_{j \beta},
$$

in the following way

$$
\omega_{(i \alpha)}=\left\langle e_{\alpha} d e_{i}\right\rangle=\sum_{j=1}^{p} \sum_{\beta=p+1}^{p+q}\left\langle e_{\alpha} \frac{\partial e_{i}}{\partial x_{j \beta}}\right\rangle d x_{j \beta}=-\left\langle e_{i} d e_{\alpha}\right\rangle=-\omega_{(\alpha i)} .
$$

The relations

$$
d \omega_{(i \alpha)}=-\sum_{j=1}^{p} \sum_{\beta=p+1}^{p+q} \omega_{(i \alpha)(j \beta)} \wedge \omega_{(j \beta)},
$$

imply the components of the corresponding curvature tensor (33) whose components of the Ricci tensor $\rho^{11}$ are given by (34).

We present now the projections of the curvature tensor for this metric which are neither considered in the framework of projective invariants nor various normalizations. More precisely we have 
Proposition 5.14. On the Grassmann manifold $\mathcal{G} r\left(V^{p \otimes q}\right)$ it exists a Riemannian metric such that $\mathcal{G} r\left(V^{p \otimes q}\right)$ is an Einstein space and the curvature tensor $R$ fulfills the relations

$$
\begin{gathered}
\left(P_{\Pi_{1}^{a}(2) \otimes \Pi_{2}^{t s}(2)} R\right)_{(i \alpha)(j \beta)(k \gamma)}{ }^{(l \nu)} \\
=\frac{1}{3}\left(\delta_{i k} \delta_{j}^{l}-\delta_{j k} \delta_{i}^{l}\right)\left(\delta_{\alpha \beta} \delta_{\gamma}^{\nu}+\delta_{\gamma \alpha} \delta_{\beta}^{\nu}+\delta_{\beta \gamma} \delta_{\alpha}^{\nu}\right) \\
\left(P_{\Pi_{1}^{t s}(2) \otimes \Pi_{2}^{a}(2)} R\right)_{(i \alpha)(j \beta)(k \gamma)}{ }^{(l \nu)} \\
=\frac{1}{3}\left(\delta_{i j} \delta_{k}^{l}+\delta_{j i} \delta_{k}^{l}+\delta_{k j} \delta_{i}^{l}\right)\left(\delta_{\alpha \gamma} \delta_{\beta}^{\nu}-\delta_{\beta \gamma} \delta_{\alpha}^{\nu}\right) \\
\left(P_{\Pi_{1}(2) \otimes \Pi_{2}(2)} R\right)_{(i \alpha)(j \beta)(k \gamma)}^{(l \nu)} \\
=\frac{1}{3}\left(\delta_{i k} \delta_{j}^{l}-\delta_{j k} \delta_{i}^{l}\right)\left(2 \delta_{\alpha \beta} \delta_{\gamma}^{\nu}-\delta_{\gamma \alpha} \delta_{\beta}^{\nu}-\delta_{\beta \gamma} \delta_{\alpha}^{\nu}\right) \\
\quad+\frac{1}{3}\left(2 \delta_{i j} \delta_{k}^{l}-\delta_{k i} \delta_{j}^{l}-\delta_{j k} \delta_{i}^{l}\right)\left(\delta_{\alpha \gamma} \delta_{\beta}^{\nu}-\delta_{\beta \gamma} \delta_{\alpha}^{\nu}\right)
\end{gathered}
$$

Proof. It follows by using straightforward computations.

Remark 5.15. Let us remark that one can obtain the Leichtweiss connection from the Akivis-Goldberg one if we put $\lambda_{(i \alpha)(j \beta)}=-\delta_{i j} \delta_{\alpha \beta}$.

We are interested in an example of a torsion-free connection $D$ on $\mathcal{G} r\left(V^{2} \otimes V^{2}\right)$, whose Ricci trace $\rho^{11}$ is symmetric, and $D$ is not the LeviCivita connection. We have in mind $\mathcal{G} r\left(V^{2} \otimes V^{2}\right)$ is homeomorphic to $\mathbb{S}_{1}^{2} \times$ $\mathbb{S}_{2}^{2}$, where $\mathbb{S}_{i}^{2}(i=1,2)$ are standard two-dimensional spheres, parametrized as

$$
x_{i}=\cos \alpha_{i} \sin \beta_{i}, \quad y_{i}=\sin \alpha_{i} \sin \beta_{i}, \quad z_{i}=\cos \beta_{i}
$$

$0<\alpha_{i}<2 \pi, 0<\beta_{i}<\pi$ (see [53] for more details).

Then we use a local coordinate system $u_{i 3}=\alpha_{i}, u_{i 4}=\beta_{i}$, and adopt computations from [10] to get

$$
\begin{aligned}
& \tilde{\Gamma}_{(i 4)(i 4)}^{(i 3)}=\tilde{\Gamma}_{(i 3)(i 3)}^{(i 3)}=\tilde{\Gamma}_{(i 4)(i 3)}^{(i 4)}=\tilde{\Gamma}_{(i 3)(i 4)}^{(i 4)}=0, \\
& \tilde{\Gamma}_{(i 4)(i 4)}^{(i 4)}=h_{i}, \quad \tilde{\Gamma}_{(i 4)(i 3)}^{(i 3)}=\cot \beta_{i}+h_{i}, \\
& \tilde{\Gamma}_{(i 3)(i 3)}^{(i 4)}=-\sin ^{2} \beta_{i}\left(\cot \beta_{i}+h_{i}\right),
\end{aligned}
$$


assuming $h_{i}=h_{i}\left(\beta_{i}\right)$ are some smooth functions, periodic with respect to $\beta_{i}$. Because of the properties of functions $h_{i}$ we see that our connection can be also extended to a global connection on $\mathbb{S}_{1}^{2} \times \mathbb{S}_{2}^{2}$.

We find the components of the Ricci trace $\tilde{\rho}^{11}=\tilde{\rho}$

$$
\begin{aligned}
& \tilde{\rho}_{(i 4)(i 4)}=\tilde{\rho}_{(i 3)(i 3)}=\frac{\partial h_{i}}{\partial \beta_{i}}+h_{i} \cot \beta_{i}-1, \\
& \tilde{\rho}_{(i 3)(i 4)}=\tilde{\rho}_{(i 4)(i 3)}=0 .
\end{aligned}
$$

One can check

$$
P_{\Pi(2) \otimes \Pi(2)} \tilde{R}=\tilde{R},
$$

and consequently, other projections vanish. Therefore, $\tilde{R}$ belongs to the simple subbundle $\Pi(2) \otimes \Pi(2)$ and $\tilde{D}$ is its corresponding connection, globally defined on $\mathcal{G} r\left(V^{2} \otimes V^{2}\right)$.

The projective transformations for this connection do not coincide with the affine transformations and this connection is not the Levi-Civita connection (see [10] for more details).

\subsection{Ricci flat connections}

We use [30], [22], [23] to define the normal projective connection $\Pi$

$$
\Pi_{(i \alpha)(j \beta)}^{(k \gamma)}=\Gamma_{(i \alpha)(j \beta)}^{(k \gamma)}-\frac{1}{p q+1}\left(\delta_{i}^{k} \delta_{\alpha}^{\gamma} \Gamma_{(m \mu)(j \beta)}^{(m \mu)}+\delta_{j}^{k} \delta_{\beta}^{\gamma} \Gamma_{(m \mu)(i \alpha)}^{(m \mu)}\right),
$$

where $\Gamma$ is an arbitrary torsion-free connection defined on $\mathcal{G} r\left(V^{p \otimes q}\right)$. The normal projective connection $\Pi$ is uniquely determined for the projective structure $P$ which consists of all projectively equivalent torsion-free connections. For $\Pi$ the corresponding Ricci curvature is

$$
\rho^{11}(\Pi)=0 .
$$

Consequently it yields

Proposition 5.16. If $\Pi$ is the normal projective connection on $\mathcal{G} r\left(V^{p \otimes q}\right)$, then its curvature belongs to the subbundle $\pi(2,1,-1)$ in the decomposition of the $\mathcal{R}\left(\mathcal{G} r\left(V^{p \otimes q}\right)\right)$.

We use [27] to prove the following theorem.

THEOREM 5.17. For the normal projective connection $\Pi$ on $\mathcal{G} r\left(V^{p \otimes q}\right)$ its curvature tensor $R(\Pi)$ belongs to $\mathcal{P}\left(\mathcal{G} r\left(V^{p \otimes q}\right)\right)$, and consequently the group of projective transformations coincides with its subgroup of affine transformations. 


\subsection{Ricci skew-symmetric connections}

As we know manifolds, that admit absolute parallelizability of directions (shortly APD manifolds) were studied by Norden [41]. He proved APD manifolds have the skew-symmetric Ricci tensor. We are now interested in do Grassmann manifolds belong to the class of APD manifolds? We answer this question affirmatively locally and non-affirmatively globally for the case $p=q=2$, i.e. for $\mathcal{G} r\left(V^{2} \otimes V^{2}\right)$. To find a torsion-free connection with the skew-symmetric curvature tensor $\rho^{11}$ defined on $\mathcal{G} r\left(V^{2} \otimes V^{2}\right)$ we recall $\mathcal{G} r\left(V^{2} \otimes V^{2}\right)$ is homeomorphic to $\mathbb{S}_{1}^{2} \times \mathbb{S}_{2}^{2}$ (see Subsection 5.4 and [53]).

Then we use a local coordinate system $u_{i 3}=\alpha_{i}, u_{i 4}=\beta_{i}, i=1,2$; $\beta_{i} \in(0, \pi)$, and adopt computations from [9] to get

$$
\begin{aligned}
& \tilde{\Gamma}_{(i 4)(i 4)}^{(i 4)}=\frac{1-\cos \beta_{i}}{\sin \beta_{i}}, \quad \tilde{\Gamma}_{(i 4)(i 4)}^{(i 3)}=-\frac{1-\cos \beta_{i}}{\sin ^{2} \beta_{i}}, \\
& \tilde{\Gamma}_{(i 3)(i 4)}^{(i 3)}=\tilde{\Gamma}_{(i 4)(i 3)}^{(i 3)}=\frac{1}{\sin \beta_{i}}, \quad \tilde{\Gamma}_{(i 3)(i 3)}^{(i 4)}=-\sin \beta_{i}, \\
& \tilde{\Gamma}_{(i 3)(i 4)}^{(i 4)}=\tilde{\Gamma}_{(i 4)(i 3)}^{(i 4)}=\tilde{\Gamma}_{(i 3)(i 3)}^{(i 3)}=1-\cos \beta_{i},
\end{aligned}
$$

where $i=1,2$. Consequently, by direct computations one can check

$$
\begin{array}{ll}
\rho_{(i 3)(i 4)}=-\rho_{(i 4)(i 3)}=R_{(14)(13)(14)}{ }^{(14)}=\sin \beta_{i}, & i=1,2, \\
\rho_{(i \alpha)(i \alpha)}=0, \quad \alpha=3,4, \quad R_{(j \beta)(k \gamma)(l \delta)}^{(m \nu)}=0, & \text { otherwise. }
\end{array}
$$

Let $c_{1}(M)$ be the first Chern class of a manifold $M$. Since $c_{1}\left(\mathbb{S}^{2}\right) \neq 0$, there exists only locally a torsion-free connection on $\mathcal{G} r\left(V^{2} \otimes V^{2}\right)$ with skewsymmetric Ricci curvature. We refer to [9] for more details. It is simple to see

$$
P_{\Pi(2) \otimes \Pi(1,1)} \tilde{R}=\tilde{R}
$$

and consequently, other projections vanish. Therefore $\tilde{R}$ belongs to the simple subbundle $\Pi(2) \otimes \Pi(1,1)$ and $\tilde{\Gamma}$ is its corresponding connection.

Remark 5.18. The connection with coefficients $\tilde{\Gamma}$ (studied in Subsections 5.4 and 5.6) can be expressed also in this way

$$
\begin{gathered}
\tilde{\Gamma}_{(j \beta)(k \gamma)}^{(i \alpha)}=\Gamma_{(j \beta)(k \gamma)}^{(i \alpha)}+\delta_{j}^{i} \delta_{\beta}^{\alpha} p_{(k \gamma)}+\delta_{k}^{i} \delta_{\gamma}^{\alpha} p_{(j \beta)}-g_{(j \beta)(k \gamma)} P^{(i \alpha)} \\
p_{(k \gamma)}=g_{(i \alpha)(k \gamma)} P^{(i \alpha)}
\end{gathered}
$$


$g$ is the standard metric of $\mathbb{S}^{2} \times \mathbb{S}^{2}$ or of $M$ in general case. The connection $\tilde{\Gamma}$ in this framework was studied by Simon in [45]. He has proved this connection is affine conformally invariant on a hypersurface of an affine space with relative normalization.

Acknowledgement. The authors express with the great pleasure their gratitude to D. V. Alekseevsky for valuable discussions to understand the problem considered in this paper and its enlightened from various point of views. We want to thank to the referee who improved this manuscript by valuable suggestions and comments. Many thanks also to: M. I. Akivis, V. Balashchenko, P. B. Gilkey, V. V. Goldberg, S. Marchiafava, V. Matveev, P. Nagy, L. Vanhecke, S. Vukmirović and L. Tamassy, for valuable discussions.

\section{REFERENCES}

[1] M. Akivis and V. Goldberg, Conformal Differential Geometry and its generalizations, Pure and Applied Mathematics, John Wiley \& Sons, New York, 1996.

[2] D. V. Alekseevsky, Riemannian spaces with unusual holonomy groups, Func. Anal and Appl., 2 (1968), 97-105.

[3] D. V. Alekseevsky, V. Cortes and C. Devchand, Yang-Mills connections over manifolds with Grassmann structure, J. Math. Phys., 44 (2003), 6047-6076.

[4] D. V. Alekseevsky and M. M. Graev, Twistors and G-structures, Izv. Ross. Akad. Nauk, Ser. Matem. Tom, 56 (1992), no. 1, 3-37; English translation: Russian Acad. Sci. Izv. Math., 40 (1993), no. 1, 1-31.

[5] D. V. Alekseevsky and S. Marchiafava, Quaternionic structures on a manifold and subordinated structures, Ann. Mat. pura Appl., CLXXI (1996), no. IV, 205-273.

[6] M. F. Atiyah, N. J. Hitchin and I. M. Singer, Self-duality in four-dimensional Riemannian geometry, Proc. Roy. Soc. London, Ser. A, 362 (1978), 425-467.

[7] T. N. Bailey and M. G. Eastwood, Complex paraconformal manifolds - their differential geometry and twistor theory, Forum Math., 3 (1991), 61-103.

[8] M. Berger, Sur les groupes d'holonomie homogène des variétés a connexion affine et des variétés riemanniennes, Bull. Soc. Math. France, 83 (1955), 279-330.

[9] N. Blažić and N. Bokan, Compact Riemann surfaces with skew-symmetric Ricci tensor, Izv. VUZ, 9 (376) (1993), 8-12.

[10] N. Blažić and N. Bokan, Projective and affine transformations of a complex symmetric connection, Bull. Austral. Math. Soc., 50 (1994), 337-347.

[11] D. Blair and A. Ledger, A characterization of oriented Grassmann manifolds, Rocky Mt. J. Math., 15 (1984), 573-584.

[12] Boerner, Representations of groups, $2^{\text {nd }}$ edition, Elsevir North-Holland, Amsterdam, 1970.

[13] N. Bokan, The decomposition theory and its applications, The math. heritage of C. F. Gauss (G. M. Rassias, ed.), World Sci. Publ. Co. Singapore (1991), pp. 66-99. 
[14] R. Bryant, Two exotic holonomies in dimension four, Path geometries, and Twistor Theory, Proc. Symp. in Pure Math., 53 (1991), 33-88.

[15] Q. S. Chi and L. Schwachhöfer, Exotic holonomy on moduli spaces of rational curves, Differ. Geom. Appl., 8 (1998), no. 2, 105-134.

[16] I. Dimitrić, A note on equivariant embeddings of Grassmannians, Publ. Inst. Math., 59 (73) (1996), 131-137.

[17] J. Dieudonné, Schur functions and group representations, Young tableaux and Schur functors in algebra and geometry, Astéerisque, 87-88 (1981), 7-19.

[18] J. Eells and S. Salamon, Constructions twistorielles des applications harmoniques, C. R. Acad. Sci. Paris Sér. I Math., 296 (1983), 685-687.

[19] W. Fulton and J. Harris, Representation Theory, GTM 129, Springer-Verlag, New York, Berlin, Heidelberg, 1991.

[20] A. Gray and L. Hervella, The sixteen classes of almost Hermitian manifolds, Ann. Mat. Pura Appl., 123 (1980), 35-58.

[21] M. Hamermesh, Group theory and its application to physical processes, Addison-Wesley Publishing Company, Reading, Massachusetts, 1962.

[22] Th. Hangan, Le pseudo-groupe projectif grassmannien (Romanian), An. Sti. Univ. Al. I. Cuza Iasi, N. Ser., Sect. Ia 11B (1965), 349-355.

[23] Th. Hangan, Tensor-product tangent bundles, Arch. der Math., 19 (4) (1968), 436-448.

[24] Th. Hangan, Sur l'intégrabilité des structures tangentes produits tensoriels réels, Ann. Mat. Pura et Appl., 126 (1980), 149-185.

[25] J. Hano and H. Ozeki, On the holonomy group of linear connections, Nagoya Math. J., 10 (1956), 97-100.

[26] C. Itzykson and M. Nauenberg, Unitary groups: Representations and Decompositions, Reviews of Modern Physics, 38 (1966), no. 1, 95-120.

[27] S. Ishihara, Groups of projective transformations and groups of conformal transformations, Jour. Math. Soc. of Japan, 9 (1957), no. 2, 195-227.

[28] G. Kempf and L. Ness, Tensor products of fundamental representations, Can J. Math., 40 (1988), 633-648.

[29] S. Kobayashi and K. Nomizu, Foundations of differential geometry Vol. 1 and 2, Interscience Publishers, New York, London, 1963.

[30] S. Kobayashi and T. Nagano, On projective connections, J. Math. Mechanics, 13 (1964), no. 2, 215-235.

[31] R. R. Kulkarni, On the Bianchi identities, Math. Ann., 199 (1972), 175-204.

[32] K. Leichtweiss, Zur Riemannschen Geometrie in Grassmannschen Mannigfaltigkeiten, Math. Zeitschr., 76 (1961), 334-366.

[33] D. E. Littlewood, Invariant theory, tensors and group characters, Phil. Trans. Royal Soc. London A, 239 (1944), 305-365.

[34] Y. Machida and H. Sato, Twistor theory of manifolds with Grassmannian structures, Nagoya Math. J., 160 (2000), 17-102.

[35] Yu. I. Manin, Gauge Field Theory and Complex Geometry, $2^{\text {nd }}$ edition, Springer-Verlag, New York, 1997. 
[36] Yu. I. Manin, New dimensions in geometry, Lect. Notes in Math., 1111, Springer-Verlag, New York (1985), pp. 59-101.

[37] S. Marchiafava, Varietà localmente grassmanniane quaternionali, Atti Accad. naz. dei Lincei, 57 (1974), 80-89.

[38] S. A. Merkulov, Paraconformal supermanifolds and nonstandard $N$-extended supergravity models, Classical Quantum Grav., 8 (1991), 557-569.

[39] S. Merkulov and L. Schwachhöfer, Classification of irreducible holonomies of torsion-free affine connections, Ann. Math. (2), 150 (1999), no. 1, 77-149.

[40] S. Merkulov and L. Schwachhöfer, Addendum to: Classification of irreducible holonomies of torsion-free affine connections, Ann. Math. (2), 150 (1999), no. 3, 1177-1179.

[41] A. P. Norden, Manifolds with affine connection, Russ. edition, Nauka, Moscow, 1976.

[42] R. Penrose, The twistor programme, Rep. Math. Phys., 12 (1977), 65-76.

[43] L. S. Pontryagin, Characteristic cycles on differentiable manifolds, Matematicheskii Sbornik (NS), 21 (63) (1947), 233-284; AMS (1950), Translation n. 32.

[44] S. Salamon, Riemannian geometry and holonomy groups, Pitman Research Notes in Mathematics Series 201, John Wiley \& Sons., New York, 1989.

[45] U. Simon, Connections and conformal structure in differential geometry, Proc. of the Conference, Brno, August 24-30, 1986, D. Reidel Publishing Company, Dordrect (1986), pp. 315-328.

[46] I. M. Singer and J. A. Thorpe, The curvature of 4-dimensional Einstein space, Global Analysis (paper in honour of K. Kodaira), Univ. of Tokyo Press, Tokyo (1969), pp. 355-365.

[47] R. Strichartz, Linear algebra of curvature tensors and their covariant derivative, Can. J. Math., XL (1988), no. 5, 1105-1143.

[48] L. Tamássy, Über den Affinzusammenhang von, zu tangentialräumen gehörenden Produkträumen, Acta Math. Acad. Sci. Hungar., 11 (1960), no. 1-2, 65-82.

[49] L. Tamássy, Aus dekomponierbaren Elementen bestehende Gebilde eines Produktraumes, Matem. Vesnik, 2 (17) (1965), 121-126.

[50] F. Tricerri and L. Vanhecke, Curvature tensors on almost Hermitian manifolds, Trans. of the Amer. Math. Soc., 267 (1981), no. 2, 365-398.

[51] S. Vukmirović, Para-quaternionic Kähler reduction, preprint (2003); arXiv: math. DG/0304424.

[52] J. A. Wolf, Space forms of Grassmann manifolds, Can. J. Math., 15 (1963), 193-205.

[53] M. I. Zelikin, Homogeneous spaces and the Riccati equation in variational calulus, Russian ed., Factorial, Moscow, 1998.

\author{
N. Bokan \\ Faculty of Mathematics \\ University of Belgrade \\ Studentski trg 16, PP 550 \\ 11001 Belgrade \\ Serbia and Montenegro \\ neda@matf.bg.ac.yu
}


P. Matzeu

Dipartimento di Matematica

Viale Merello 92

09123 Cagliari

Italy

matzeu@vaxca1.unica.it

Z. Rakić

Faculty of Mathematics

University of Belgrade

Studentski trg 16, PP 550

11001 Belgrade

Serbia and Montenegro

zrakic@matf.bg.ac.yu 